\title{
Revista Colombiana de

\section{Consenso de expertos sobre el manejo clínico de la hipertensión arterial en Colombia. Sociedad Colombiana de Cardiología y Cirugía}

\author{
Luis Moya a,b ${ }^{\mathrm{a}}$, Javier Moreno ${ }^{\mathrm{a}, \mathrm{c}}$, Manuel Lombo ${ }^{\mathrm{a}}$, Carlos Guerrero ${ }^{\mathrm{a}, \mathrm{d}}$, \\ Dagnóvar Aristizábal $^{\mathrm{e}}$, Adolfo Vera ${ }^{\mathrm{b}, \mathrm{f}}$, Enrique Melgarejo ${ }^{\mathrm{b}}$, Jhon Conta ${ }^{\mathrm{h}}$, \\ Carlos Gómez ${ }^{\mathrm{g}}$, Diana Valenzuela ${ }^{i}$, Mauricio Ángel ${ }^{i}$, Heriberto Achury ${ }^{j}$, \\ Rubén Duque $^{k}$, Ángela Triana', Julián Gelves ${ }^{a, d}$, Alfonso Pinzón ${ }^{a}$, \\ Alberto Caicedo ${ }^{\mathrm{g}, \mathrm{h}}$, César Cuéllar ${ }^{\mathrm{m}}$, Jorge Sandoval ${ }^{\mathrm{n}}$, Juan Pérez ${ }^{\mathrm{a}}$, \\ Alejandro Rico-Mendoza ${ }^{\circ}$ y Alexandra Porras-Ramírez ${ }^{\circ, *}$
}

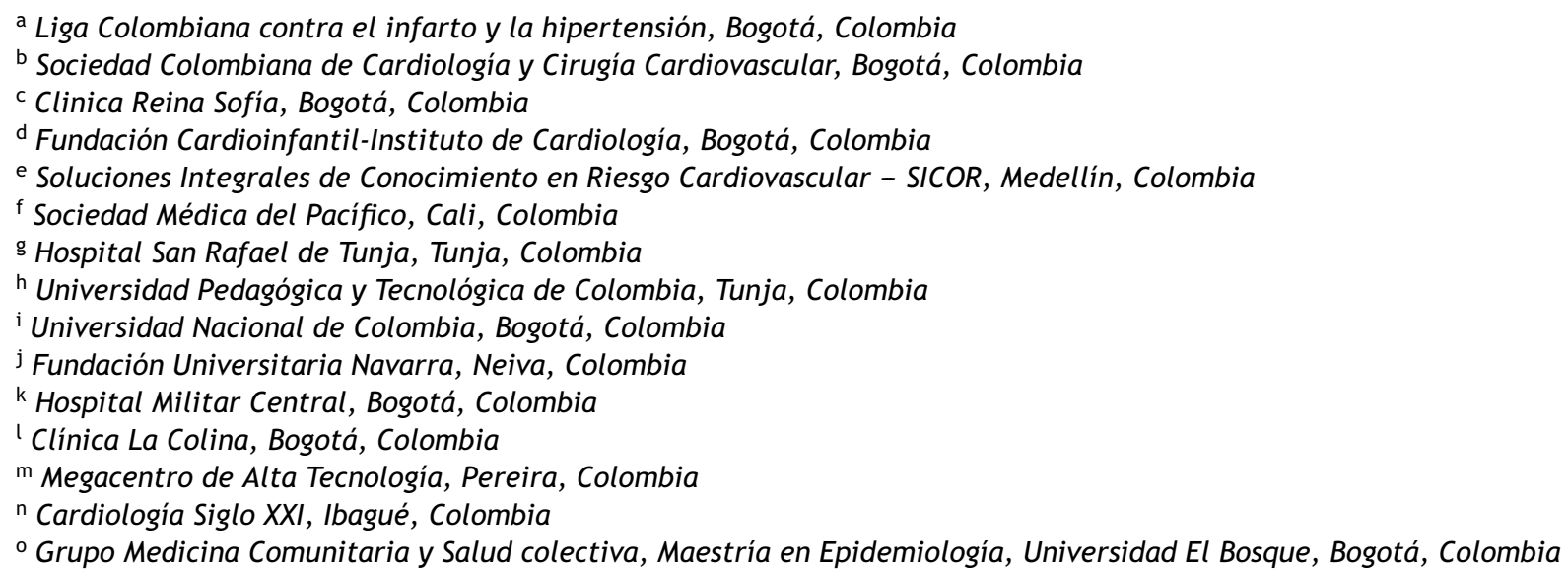

Recibido el 11 de agosto de 2018; aceptado el 27 de septiembre de 2018

Disponible en Internet el 7 de noviembre de 2018

PALABRAS CLAVE

Hipertensión arterial;

Consenso;

Enfermedad

cardiovascular
Resumen De manera paralela, a raíz de la nueva evidencia científica que respalda el tratamiento intensivo de la hipertensión arterial, en 2017 el Colegio Americano de Cardiología (ACC) y la Asociación Americana del Corazón (AHA) establecieron nuevas directrices para la prevención, detección, evaluación y manejo de la hipertensión arterial en adultos.

\footnotetext{
* Autor para correspondencia.

Correo electrónico: porras.alexandra@gmail.com (A. Porras-Ramírez).
} 


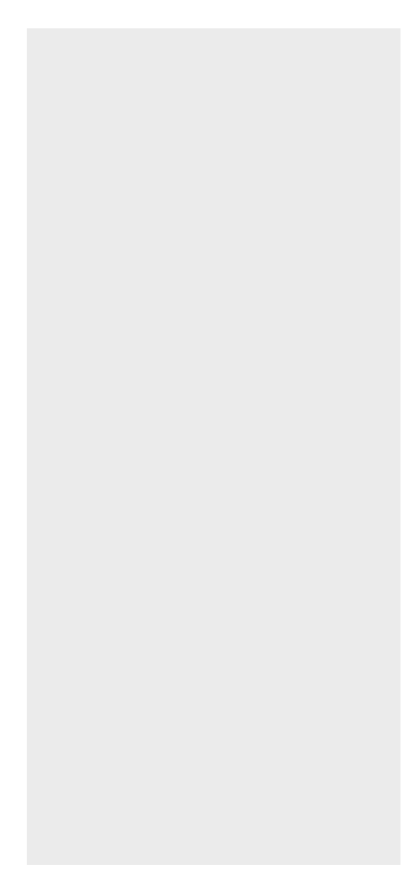

\section{KEYWORDS}

Arterial hypertension; Consensus; Cardiovascular disease

\section{Introducción}

La hipertensión es una de las causas principales de enfermedad cardiovascular y muerte prematura en todo el mundo ${ }^{1-3}$. its intensive treatment. hypertensive. as sick.
La recomendación formal fue utilizar menores umbrales de presión arterial para el inicio del tratamiento antihipertensivo. Esta consideración se derivó de la existencia de estudios que asociaban el logro de cifras menores de presión arterial con la disminución significativa en el riesgo de enfermedad cardiovascular y de la mortalidad en la población hipertensa.

A la luz de esta nueva definición de hipertensión arterial, Muntner et al. consideran que su prevalencia en los Estados Unidos sería 45,6\%, comparado con el 31,9\%, si se usaran los parámetros previamente aceptados e implementados desde el JNC7, comité en el que el porcentaje de adultos estadounidenses recomendados para tratamiento antihipertensivo pasaría de $34,3 \%$ a $36,2 \%$, en tanto que el porcentaje de hipertensos que no logran las metas establecidas se incrementaría de 39 a 53,4\%.

Por su parte, Bundy et al. llegaron a conclusiones similares, calculando que $45,4 \%$ de los adultos estadounidenses (105.3 millones) mayores de 20 años tendrían hipertensión según la nueva clasificación, comparado con los datos del año 2014, que consideraban como hipertensos al 32,0\% (74.1 millones). Esta diferencia de 13,5\% equivale a 31.3 millones de personas que serían clasificadas como hipertensas.

En Colombia, la aplicación de la nueva clasificación supondría pasar de una prevalencia de $22,0 \%$ en 2016 a un valor estimado del $43,3 \%$ en la actualidad, tan solo por variar la cifra de corte para ser considerado hipertenso, lo cual es equivalente a catalogar como enfermos a aproximadamente 21 millones de personas.

(c) 2018 Sociedad Colombiana de Cardiología y Cirugía Cardiovascular. Publicado por Elsevier España, S.L.U. Este es un artículo Open Access bajo la licencia CC BY-NC-ND (http:// creativecommons.org/licenses/by-nc-nd/4.0/).

\section{Expert consensus on the clinical management of arterial hypertension in Colombia [Colombian Society of Cardiology and Cardiovascular Surgery]}

Abstract In 2017, the American College of Cardiology (ACC) and the American Heart Association (AHA) concurrently established new guidelines for the prevention, detection, assessment and management of arterial hypertension in adults, based on new scientific evidence supporting

The formal recommendation was to use lower arterial pressure thresholds for initiating antihypertensive treatment. This consideration arose from studies which related the achievement of lower arterial pressure figures with a significant decrease in the risk of cardiovascular disease and mortality in the hypertensive population.

In light of this new definition of arterial hypertension, Muntner et al. consider that the prevalence of hypertension in the United States would be $45.6 \%$, compared with $31.9 \%$ if the previously accepted and implemented parameters from the JNC7 were applied. According to this committee, the percentage of American adults recommended for antihypertensive treatment would go from $34.3 \%$ to $36.2 \%$, while the percentage of hypertensives not achieving the established goals would increase from 39 to $53.4 \%$.

Meanwhile, Bundy et al. reached similar conclusions, calculating that $45.4 \%$ of adult Americans over the age of 20 (105.3 million) would have hypertension according to the new classification, compared to data from 2014, which considered $32.0 \%$ (74.1 million) to be hypertensive. This $13.5 \%$ difference amounts to 31.3 million people who would be classified as

In Colombia, the application of the new classification would mean going from a prevalence of $22.0 \%$ in 2016 to an estimated $43.3 \%$, currently, just by changing the cut-off value for being considered hypertensive, which would amount to classifying approximately 21 million people

(c) 2018 Sociedad Colombiana de Cardiología y Cirugía Cardiovascular. Published by Elsevier España, S.L.U. This is an open access article under the CC BY-NC-ND license (http:// creativecommons.org/licenses/by-nc-nd/4.0/). 
adecuado, el impacto hemodinámico sobre órganos como corazón, riñón y cerebro causa un grave deterioro funcional y acarrea consecuencias en la calidad de vida, así como secuelas irreversibles.

En Colombia, como sucede en otras latitudes, se ha visto un incremento en la prevalencia de hipertensión arterial (hipertensión arterial). El documento de análisis de situación en salud realizado por el Ministerio de Salud y Protección Social del año 2016, informa una prevalencia del 22\% entre la población general. Una de las explicaciones posibles de este aumento ha sido la migración constante de grupos poblacionales del área rural al área urbana, lo cual conduce a cambios importantes en los comportamientos de salud y al aumento de los factores de riesgo cardiovascular. En Colombia la problemática es particular ya que la diversidad de razas, climas y costumbres, la estructura del sistema de salud con sus políticas de prevención que han sido insuficientes, sumado al nivel sociocultural tan variado y a las altas tasas de pobreza, hacen que nuestra población sea diferente de las poblaciones europeas y norteamericanas. Estos factores, junto con los fenómenos poblacionales ya mencionados, generan aumento muy particular en la carga de factores de riesgo cardiovascular que diferencia a Colombia de otras regiones.

De manera paralela, a raíz de la nueva evidencia científica que respalda el tratamiento intensivo de la hipertensión arterial, en 2017 el Colegio Americano de Cardiología (ACC) y la Asociación Americana del Corazón (AHA) establecieron nuevas directrices para la prevención, detección, evaluación y manejo de la hipertensión arterial en adultos. La recomendación formal fue utilizar menores umbrales de presión arterial para el inicio del tratamiento antihipertensivo ${ }^{5-7}$. Esta consideración se derivó de la existencia de estudios que asociaban el logro de cifras menores de presión arterial con la disminución significativa en el riesgo de enfermedad cardiovascular y de la mortalidad en la población hipertensa. A la luz de esta nueva definición de hipertensión arterial, Muntner et al. ${ }^{8}$, consideran que la prevalencia de hipertensión en los Estados Unidos sería $45,6 \%$ (comparado con el $31,9 \%$ ), si se usaran los parámetros previamente aceptados e implementados desde el JNC7, comité en el cual el porcentaje de adultos estadounidenses recomendados para tratamiento antihipertensivo pasaría de 34,3 a $36,2 \%$, y el porcentaje de hipertensos que no logran las metas establecidas se incrementaría de 39 a $53,4 \%$.

Bundy et al. ${ }^{9}$, llegan a conclusiones similares calculando que $45,4 \%$ de los adultos estadounidenses (105.3 millones) mayores de 20 años tendrían hipertensión según la nueva clasificación, comparado con los datos de 2014, que consideraban como hipertensos al 32,0\% (74.1 millones). Esta diferencia de $13,5 \%$ equivale a 31.3 millones de personas que serían clasificadas como hipertensas.

En Colombia, la aplicación de la nueva clasificación supondría pasar de una prevalencia de $22,0 \%$ en 2016 a un valor estimado del $43,3 \%$ en la actualidad, tan solo por variar la cifra de corte para ser considerado hipertenso, lo cual es equivalente a considerar como enfermos a aproximadamente 21 millones de personas.

$\mathrm{Si}$ bien las directrices han sido respaldadas por muchas otras sociedades profesionales, la Academia Estadounidense de Médicos de Familia (AAFP) y el Colegio Estadounidense de
Médicos (ACP) se han negado a dar su apoyo. Sus principales objeciones se refieren al objetivo terapéutico de lograr cifras de presión arterial sistólica menores a $130 \mathrm{~mm} / \mathrm{Hg}$. Las preocupaciones específicas se basan, especialmente, en el riesgo de intentar estas metas estrictas en pacientes ancianos con hipertensión. La AAFP y la ACP afirman que el comité de directrices de ACC/AHA dio demasiado peso al ensayo SPRINT y no prestó suficiente atención a la revisión sistemática de otras pruebas. También hacen especial énfasis en que los daños, los costos y la complejidad de la atención asociada con el nuevo objetivo no justifican los presuntos beneficios. Por tanto, la ACP y AAFP han publicado sus propias pautas para adultos mayores (mayores de 60 años) que recomiendan una presión sistólica objetivo inferior a $150 \mathrm{~mm} / \mathrm{Hg}$, bajando el valor a $140 \mathrm{~mm} / \mathrm{Hg}$ en personas seleccionadas con alto riesgo cardiovascular, siempre y cuando exista una adecuada monitorización de los posibles efectos adversos derivados. Concluyen en su revisión que debe haber equilibrio óptimo entre beneficios y daños. Por ello respaldan las pautas establecidas en 2014 por el Octavo Comité Nacional Conjunto (JNC 8).

Ante este panorama y teniendo las mismas inquietudes, la Sociedad Colombiana de Cardiología y Cirugía Cardiovascular revisó las directrices propuestas por el ACC y la AHA a fin de evaluar cómo podrían aplicarse al sistema de salud colombiano.

Se realizó un consenso de expertos de la Sociedad basado en la revisión exhaustiva de la literatura médica con énfasis en el manejo farmacológico y no farmacológico de la hipertensión arterial, ajustado a la población colombiana. El objetivo era proporcionar a los médicos propuestas prácticas que pudieran conducir a mejorar la atención del paciente con hipertensión arterial y pronunciarse frente a las nuevas metas planteadas por las Guías del 2017 del ACC y de la AHA ya mencionadas.

En Colombia, el consenso fue alcanzado en todas las declaraciones a través de una ronda múltiple de revisión y proceso de votación Delphi modificado donde se requirió un $80 \%$ de acuerdo para incluir una recomendación consensuada. La modificación del método Delphi consistió en usar el método denominado "político" que fue propuesto en la década de 1970 cuyo objetivo no es que un grupo tome una decisión, sino que un grupo de expertos presenten todas las opciones posibles ante un problema*. El objetivo es analizar un problema y generar opciones. Las declaraciones se basaron en la opinión experta de los participantes teniendo en cuenta la revisión crítica de literatura.

Como método de calificación se usó el descriptivo, calculando como medida de tendencia central la mediana de las calificaciones e identificando los valores extremos del rango de respuestas. Si la mediana medida estaba en un rango de 7 a 9 se consideraría como una recomendación adecuada o de primera línea y para este consenso calificada como la Altamente recomendado. Si la mediana se ubicaba en un rango de 1 a 3 se tomaría como una recomendación clasificada como III- recomendadas solo si el criterio clínico ve utilidad. Finalmente, si la mediana se ubicaba entre 4 y 6 se

\footnotetext{
* Ampliar información en Meyrick J. The Delphi method and health research; Health Education, 103(1):7-16, https://doi.org/10.1108/09654280310459112
} 
consideraría que la recomendación se clasificaría como Ilbrecomendado. Como método estadístico se usó la prueba de significancia de Mann-Whitney con el fin de estimar un valor de $p$, que de hallarse por debajo de 0,05 se consideraría estadísticamente significativo ${ }^{10}$.

\section{Preguntas priorizadas por el grupo de expertos}

El grupo elaboró un listado de 14 preguntas orientadoras a saber:

1 ¿Nos confunde o nos alerta la nueva clasificación de hipertensión arterial?

2 ¿Qué escala de clasificación de riesgo cardiovascular es aplicable a la población colombiana adulta?

3 ¿Qué ayudas paraclínicas son fundamentales para el inicio y seguimiento del hipertenso en nuestro medio?

4 ¿Cuáles deben ser las estrategias de manejo no farmacológico y preventivo implementadas en pacientes adultos con hipertensión en Colombia?

5 ¿Cuál debe ser el manejo farmacológico inicial de la hipertensión arterial, acorde con las características del sistema de salud y la diversidad de la población colombiana?

6 ¿A qué tipo de pacientes se le debe suministrar terapia combinada? ¿Qué medicamentos se deben usar en combinación?

7 ¿Cuál debe ser el esquema básico de seguimiento aplicable a los pacientes adultos con hipertensión en Colombia, según nivel de riesgo y enfermedades preexistentes (enfermedad coronaria, insuficiencia cardíaca congestiva, fibrilación auricular, enfermedad cerebrovascular, insuficiencia renal crónica, diabetes mellitus, cirrosis, obesidad, trasplante)?

8 ¿Cuáles son las indicaciones de uso del ecocardiograma en el seguimiento del paciente adulto con hipertensión arterial?

9 ¿Cuál es el papel de las terapias alternativas disponibles en Colombia en el tratamiento de la hipertensión arterial? ¿Cuál es la evidencia de su eficacia en pacientes con hipertensión arterial?

10 ¿Cuál debe ser el manejo de la hipertensión arterial en edades especiales (niños, jóvenes y ancianos)?

11 ¿Qué debe saber el médico sobre hipertensión arterial secundaria?

12 ¿Cuál es la indicación actual de manejo intervencionista y cuáles son aplicables en Colombia?

13 ¿Cómo debe ser la valoración prequirúrgica y el manejo perioperatorio del paciente hipertenso?

14 ¿Cuál es el tratamiento de la hipertensión en el adulto mayor?

\section{Resultados del consenso}

A continuación, y por cada pregunta, se presentan los resultados del consenso de expertos.

\section{Pregunta 1 ¿Nos confunde o nos alerta la nueva} clasificación de hipertensión arterial?

- La nueva clasificación nos confunde, por lo que recomendamos NO adoptarla y continuar el punto de corte igual a como se definió en la guía de práctica clínica para Colombia en el año 2013. Es decir, hipertensión arterial se define cuando las cifras tensionales son iguales o mayores que $140 / 90 \mathrm{~mm} \mathrm{Hg}$ (Ilb- recomendado) (recomendación adecuada o de primera línea).

- En cuanto a la meta de tratamiento general, proponemos mantener un objetivo de cifras de menos de $140 / 90 \mathrm{~mm}$ $\mathrm{Hg}$. En la población mayor de 70 años la meta de presión arterial sistólica será de menos de $140 \mathrm{~mm} \mathrm{Hg}$ siempre y cuando la presión diastólica no sea menor de $80 \mathrm{~mm}$ $\mathrm{Hg}$. Si existen efectos colaterales o fragilidad, considerar una meta más laxa de $150 / 90 \mathrm{~mm} \mathrm{Hg}$ (Ilb-recomendado) (recomendación adecuada o de primera línea) ${ }^{11-13}$.

- En la nueva clasificacion valoramos la importancia que se da a la estratificación cardiovascular y se debe adoptar una escala de riesgo validada en cada población para así definir metas de tratamiento. Para nuestro medio consideramos dos métodos de estratificación de riesgo, que se resumen en la pregunta 2 .

- Existe suficiente evidencia para afirmar que una meta de tratamiento farmacológico de $130 \mathrm{~mm} \mathrm{Hg} / 80 \mathrm{~mm} \mathrm{Hg}$ es válida para población hipertensa de alto riesgo cardiovascular (la-Altamente recomendado) (recomendación adecuada o de primera línea) $)^{14,5,15}$.

- En pacientes diabéticos hubo una reducción del 51,0\% en los eventos cardiovasculares. Esto se basó en la comparación de dos grupos de pacientes diabéticos, uno con una meta de cifras tensionales diastólicas menores o iguales a $90 \mathrm{~mm} \mathrm{Hg}$ y otro con cifras tensionales menores o iguales a $80 \mathrm{~mm} \mathrm{Hg}$. Este estudio indicó la necesidad de considerar metas diferentes en función del nivel de riesgo cardiovascular (Ib-Altamente recomendado) (recomendación adecuada o de primera línea ${ }^{16-18}$.

- Hay menor incidencia de eventos cardiovasculares con un valor de presión arterial diastólica de $82,6 \mathrm{~mm} \mathrm{Hg}$ (lbAltamente recomendado) (recomendación adecuada o de primera línea) ${ }^{19}$.

- La tasa más baja de mortalidad se obtuvo con cifras tensionales menores o iguales de $86,5 \mathrm{~mm} \mathrm{Hg}$ (lb-Altamente recomendado) (recomendación adecuada o de primera línea $)^{20}$.

- Para población mayor de 70 años la obtención de esta meta $(130 / 80 \mathrm{~mm} \mathrm{Hg})$ frecuentemente conlleva efectos adversos significativos que contrarrestan los beneficios cardiovasculares esperados y se requiere vigilar estrechamente estos pacientes (la-Altamente recomendado) (recomendación adecuada o de primera línea) ${ }^{19}$.

- Se definen dos estadios de hipertensión arterial: estadio I a todo paciente con tensiones arteriales entre $140-159 \mathrm{~mm}$ $\mathrm{Hg}$ la sistólica y/o 90 - $99 \mathrm{~mm} \mathrm{Hg}$ la diastólica. Se interpretará como estadio II a todo paciente con tensión arterial mayor o igual a $160 \mathrm{~mm} \mathrm{Hg}$ y/o diastólica mayor o igual a 
100 mm Hg (la-Altamente recomendado) (recomendación adecuada o de primera línea) ${ }^{19}$.

- Se considera en riesgo de desarrollo de hipertensión arterial a todo paciente por lo demás sano (de bajo riesgo o de riesgo intermedio cardiovascular) con más de dos tomas en consulta con cifras de tensión arterial sistólica entre $130-140 \mathrm{~mm} \mathrm{Hg}$ y tensión arterial diastólica $80-90 \mathrm{~mm} \mathrm{Hg}$, haciendo énfasis en que se debe empoderar a estos pacientes de su salud mediante la promoción de las actividades de autocuidado y la prevención no farmacológica (la-Altamente recomendado) (recomendación adecuada o de primera línea) ${ }^{19}$.

- Nos alerta la recomendación de AHA/ACC de buscar metas más estrictas. Toda meta impuesta por el médico debe ser seguida con el fin de disminuir las complicaciones de la hipertensión arterial. De igual manera, se debe realizar valoración de riesgo cardiovascular antes de iniciar cualquier manejo terapéutico antihipertensivo (la-Altamente recomendado) (recomendación adecuada o de primera línea).

\section{Pregunta 2 ¿Qué escala de clasificación de riesgo cardiovascular es aplicable a la población colombiana adulta?}

- El consenso considera que la escala de Framingham sobreestima el riesgo en la población colombiana ${ }^{21}$. La escala PROCAM convencional sobreestima el riesgo en pacientes de alto riesgo y tiene una capacidad de discriminación baja (ROC menor de 0,7). La escala PROCAM por sexo discrimina adecuadamente bajo riesgo y riesgo intermedio, pero no ocurre igual en pacientes de alto riesgo (III-recomendadas solo si el criterio clínico ve utilidad).

- Se deben validar las escalas AHSACC2013, ACCVD y SCORE en Colombia (Ib-Altamente recomendado) (recomendación adecuada o de primera línea).

- Dado el contexto anterior, este consenso recomienda usar el conteo de factores de riesgo clásicos, considerando como alto riesgo pacientes con dos o más factores de riesgo. Para la cuantificación del riesgo a 10 años se podría utilizar la escala INTERHEART, que, aunque evalúa el riesgo de infarto, ha mostrado un buen desempeño e incluye pacientes colombianos ${ }^{22,23}$ (Ib-Altamente recomendado) (recomendación adecuada o de primera línea).

\section{Pregunta 3 ¿Qué ayudas paraclínicas son fundamentales para el inicio y seguimiento en el paciente hipertenso en Colombia?}

- En toda persona mayor a 3 años se recomienda conocer el valor de su presion arterial.

- Se debe realizar tamización de presión arterial a toda persona menor de 50 años mediante tomas ocasionales (al menos una vez al año) de presión arterial (lb-Altamente recomendado) (recomendación adecuada o de primera línea $)^{24}$.

- Se debe realizar tamización activa de toda persona mayor de 50 años con tomas de presión arterial al menos cada 3 meses (lb-Altamente recomendado) (recomendación adecuada o de primera línea $)^{24}$.
- En personas con diagnóstico y tratamiento de hipertensión arterial, se debe evaluar el daño en órgano blanco mendiante cuadro hemático, perfil lipídico, electrolitos TSH, creatinina, calcio, sodio, potasio, parcial de orina con microalbuminuria, ácido úrico y electrocardiograma, al menos una vez cada año. El ecocardiograma se reversa a criterio clínico o cuando se sospeche compromiso de órgano blanco (Ib-Altamente recomendado) (recomendación adecuada o de primera línea) $)^{5,25,26}$.

- La frecuencia de la evaluación mediante laboratorios en individuos con diagnóstico y tratamiento de hipertensión arterial dependerá de los factores de riesgo que tenga (IbAltamente recomendado) (recomendación adecuada o de primera línea).

- Se debe recomendar al paciente la medición y el registro sistemáticos de su presión arterial en casa bajo el esquema AMPA (tomas diarias, diurna y nocturna, de la presión arterial durante seis días consecutivos o más) en las siguientes situaciones:

- Como seguimiento del paciente con diagnóstico de hipertensión arterial.

- Cuando se sospeche hipertensión arterial de bata blanca.

- Cuando se sospeche no adherencia al tratamiento.

- Cuando hay daño de órgano blanco o aumento del riesgo global, aunque tenga cifras tensionales adecuadas en la consulta ${ }^{5,27}$ (Ib-Altamente recomendado) (recomendación adecuada o de primera línea).

Se recomienda realizar monitorización ambulatoria de la presión arterial de 24 horas (MAPA) mediante método estándar ${ }^{5,10,17,27}$, en los siguientes casos:

- Presión arterial en el consultorio con cifras sistólicas entre 130 y 160 y diastólicas entre 80 y $100 \mathrm{~mm} \mathrm{Hg}$.

- Adultos con terapia farmacológica antihipertensiva múltiple con mediciones durante la consulta de $10 \mathrm{~mm} \mathrm{Hg}$ por encima de la meta.

- Para tamización de "hipertensión de bata blanca" (alternativamente podría apelarse a la medición domiciliaria ya descrita, según disponibilidad) ${ }^{27,28}$.

- Cuando se haga modificación del tratamiento en pacientes con metas de presión arterial no alcanzadas, especialmente en presencia de hipertensión nocturna por considerarse que hay aumento del riesgo cardiovascular (Ib-Altamente recomendado) (recomendación adecuada o de primera línea).

- Cuando exista riesgo cardiovascular incrementado según los parámetros de INTERHEART ya mencionados ${ }^{5}$.

- Se debe dotar de equipo de monitorización de tensión arterial a los programas de hipertensión arterial (IbAltamente recomendado) (recomendación adecuada o de primera línea $)^{29-31}$.

- Se deben tener en cuenta todas las posibles presentaciones clínicas de la enfermedad, incluyendo hipertensión arterial sistólica en el adulto joven, hipertensión arterial diastólica en edad media e hipertensión arterial sistólica aislada del adulto mayor, entre otras, para el enfoque adecuado e integral del paciente hipertenso (IbAltamente recomendado) (recomendación adecuada o de primera línea) $)^{5,32}$. 


\section{Pregunta 4 ¿Cuáles deben ser las estrategias de manejo no farmacológico y preventivo implementadas en pacientes adultos con hipertensión arterial?}

- El tratamiento no farmacológico es la base de cualquier terapia para el tratamiento de la hipertensión arterial y no reemplaza el manejo farmacológico sino la complementa (lla-recomendado) (recomendación adecuada o de primera línea $)^{5,63,64}$.

- Se recomienda promover el manejo de pacientes con diagnóstico de hipertensión arterial por grupos interdisciplinarios (Ila-recomendado) (recomendación adecuada o de primera línea) ${ }^{5,63,64}$.

- Se recomienda el estilo o patrón de dieta DASH (del inglés Dietary Approaches to Stop Hypertension) que recomienda incluir altas cantidades de granos enteros, frutas, verduras y legumbres, nueces, así como, la ingesta de cantidades moderadas de lácteos bajos en grasa y cantidades bajas de productos procesados ricos en grasas totales y saturadas, sodio y bebidas endulzadas; está asociada con la prevención adecuada y el tratamiento de pacientes con presión arterial alta (defendidas por la AHA) (la-Altamente recomendado) (recomendación adecuada o de primera línea) $)^{5,65-69}$.

- Se recomienda seguir siempre un patrón dietético saludable que debe prescribirse para la prevención y tratamiento de algunas otras enfermedades crónicas, que incluyen tanto dislipidemia e hiperglucemia que junto con la hipertensión arterial son factores de riesgo independientes para el desarrollo de enfermedad renal crónica y eventos cardiovasculares mayores (lb-Altamente recomendado) (recomendación adecuada o de primera línea) ${ }^{5,65-69}$.

- La interacción entre el estado hipertensivo y la dieta fue estadísticamente significativa para la presión arterial sistólica $5,5 \mathrm{~mm} \mathrm{Hg}$ (p 0,001) y marginalmente significativa para la presión arterial diastólica $3 \mathrm{~mm} \mathrm{Hg}(\mathrm{p} \mathrm{0,03)} \mathrm{(la-}$ Altamente recomendado) (recomendación adecuada o de primera línea) $)^{5,65-69}$.

- La respuesta individual a la sal es mayor en pacientes de raza negra, mujeres, diabéticos y ancianos, así que específicamente en estos pacientes debe recomendarse la disminución de sal (la-Altamente recomendado) (recomendación adecuada o de primera línea) ${ }^{5,65-69}$.

- Los beneficios cardiovasculares de la ingesta reducida de sal están a la par con los beneficios de las reducciones en toda la población del consumo de tabaco, la obesidad y la actividad física regular (Ib-Altamente recomendado) (recomendación adecuada o de primera línea) ${ }^{5,65-69}$.

- Es necesario incluir como política pública nacional a las grandes cadenas productoras de alimentos para que reduzcan el contenido de sal en los alimentos más consumidos por la población general, por ejemplo, pan, productos cárnicos, sopas y cereales de desayuno. Además, se debe etiquetar adecuadamente los productos altos en contenido de sal y evitar la transmisión de publicidad engañosa que invite a los más jóvenes a consumir estos alimentos procesados, azucarados y con alto contenido de grasas saturadas (Ib-Altamente recomendado) (recomendación adecuada o de primera línea) ${ }^{5,65-69}$.
- Con miras a reducir el sodio en la alimentación, se recomienda lo siguiente a la población general (lb-Altamente recomendado) (recomendación adecuada o de primera línea $)^{5,65-69}$ :

Prefiera el consumo de alimentos de origen natural, compre más verduras y frutas frescas y preferentemente lácteos descremados.

- Elija el consumo de carnes frescas, pollo, pescado en lugar de aquellas conservadas con sal, y cocine estos con un mínimo de sal.

- Consuma con moderación alimentos marinados a los cuales se les adiciona sal.

- Si consume alimentos procesados, envasados, prefiera los reducidos en sodio.

- Compare las etiquetas de información nutricional de los alimentos, al emplear caldos, bases, sopas deshidratadas (de sobre); no adicione sal.

- Retire el salero de la mesa.

- No adicione sal a la comida de bebés y niños pequeños, ellos perciben los sabores de forma distinta y por ello no necesitan agregar sal.

- Modere el consumo de alimentos conservados, salmueras, embutidos y quesos muy salados.

- Prefiera el uso de condimentos y especias naturales.

- Modere el consumo de alimentos que contengan polvo de hornear (bicarbonato de sodio) como tortas y bizcochos.

- Pruebe primero sus alimentos antes de usar salero; evite usar sal al tanteo, use un instrumento de medida eje, cucharita.

- La Organización Mundial de la Salud -OMS-, recomienda en adultos una ingesta diaria de potasio procedente de la comida de $90 \mathrm{mmol}(3.500 \mathrm{mg})$, los cuales pueden obtenerse de consumir de 4 a 5 porciones diarias de frutas y verduras, pescados, frutos secos y lácteos entre otros (lbAltamente recomendado) (recomendación adecuada o de primera línea) $)^{5,65-69}$.

- Hay evidencia Clase I, Nivel B que indica que 150 minutos de actividad física semanal ofrecen una alternativa que puede usarse para complementar la medicación antihipertensiva (Ib-Altamente recomendado) (recomendación adecuada o de primera línea $)^{70}$.

- Una reducción equivalente al $50 \%$ del consumo de tabaco entre adultos, reduciría en un tercio las muertes evitables relacionadas con éste (la-Altamente recomendado) (recomendación adecuada o de primera línea) ${ }^{5,65-69}$.

- Reducir el consumo de alcohol se asoció con una reducción significativa de la presión arterial sistólica y diastólica media de 3,31 mm Hg (IC 95\% mayor o igual 2,52 a mayor o igual $4,10 \mathrm{~mm} \mathrm{Hg}$ ) y mayor o igual $2,04 \mathrm{~mm} \mathrm{Hg}$ (IC 95\% mayor o igual 1,49 a mayor o igual $2,58 \mathrm{~mm} \mathrm{Hg}$ ), respectivamente. Se observó una relación dosis-respuesta entre el porcentaje medio de reducción de alcohol y la reducción media de la presión sanguínea. Los efectos de la intervención se mejoraron en aquellos con una presión arterial basal más alta (la-Altamente recomendado) (recomendación adecuada o de primera línea) ${ }^{5,65-69}$.

- El compromiso entre médico y paciente, en educación sobre su enfermedad y complicaciones, concientiza y responsabiliza al paciente e incide en las tasas de adherencia 
y en la disminución de eventos mayores (infarto de miocardio, insuficiencia renal, accidente cerebrovascular). Como política del Capítulo de hipertensión arterial de la Sociedad Colombiana de Cardiología y Cirugía Cardiovascular, se sugiere emprender campañas de paciente responsable (la-Altamente recomendado) (recomendación adecuada o de primera línea) ${ }^{5,65-69}$.

\section{Pregunta 5 ¿Cuál debe ser el manejo farmacológico inicial de la hipertensión arterial, acorde con las características del sistema de salud y la diversidad de la población colombiana?}

- Existen distintos grupos de fármacos que se consideran como de primera línea para el tratamiento farmacológico de la hipertensión arterial: diuréticos beta-adrenérgicos, inhibidores de la enzima de conversión de la angiotensina (IECA), bloqueadores de los canales de calcio, antagonista de los receptores de la angiotensina II (ARA II) y bloqueadores alfa. La preferencia en la utilización en cada uno de dichas familias, debe establecerse en función de diversos parámetros, tales como costo, enfermedades asociadas, efectividad, tolerancia, efectos esperados, impacto sobre la calidad de vida (Ib-Altamente recomendado) (recomendación adecuada o de primera línea) ${ }^{5,33}$.

- Se recomienda al iniciar tratamiento farmacológico el uso de un medicamento tiazídico si no hay contraindicación o clortalidona o indapamida (lb-Altamente recomendado) (recomendación adecuada o de primera línea) $)^{33,34}$. Al iniciar el tratamiento antihipertensivo con diurético, debe valorarse el inicio con tiazidas versus un diurético similar a la tiazida, como clortalidona $(12,5-25,0 \mathrm{mg}$ una vez al día) o indapamida (1,5 mg de liberación modificada una vez al día o 2,5 mg una vez al día) (Ib-Altamente recomendado) (recomendación adecuada o de primera línea) ${ }^{33,34}$.

- Para los pacientes que ya están recibiendo tratamiento con hidroclorotiazida y cuya presión arterial es estable y está bien controlada, se continúa el tratamiento con este medicamento (lb-Altamente recomendado) (recomendación adecuada o de primera línea) $)^{33,34}$.

- Los IECA como opción alterna de inicio de tratamiento son una herramienta tan adecuada como los diuréticos, aunque la evidencia no es tan fuerte como la de los anteriores. Sin embargo, estos tienen un alto valor en la disminución del impacto de la hipertensión arterial en órgano blanco. Los ARA II deben considerarse solo en aquellos pacientes que han tenido efectos adversos relacionados con el uso de los IECA (lb-Altamente recomendado) (recomendación adecuada o de primera línea) ${ }^{33,34}$.

- Al iniciar el tratamiento antihipertensivo con diurético, debe valorase el inicio con tiazidas versus un diurético similar a la tiazida, como clortalidona $(12,5-25,0 \mathrm{mg}$ una vez al día) o indapamida (1,5 mg de liberación modificada una vez al día o $2,5 \mathrm{mg}$ una vez al día) (Ib-Altamente recomendado) (recomendación adecuada o de primera línea) $)^{33,34}$.

- Para las personas que ya están recibiendo tratamiento con hidroclorotiazida y cuya presión arterial es estable y está bien controlada, continuar el tratamiento con este medicamento (Ib-Altamente recomendado) (recomendación adecuada o de primera línea $)^{5,33,34}$.

- Iniciar tratamiento con medicamentos antihipertensivos a personas menores de 70 años con hipertensión en etapa 1 que tengan uno o más de los siguientes: daño de órgano blanco, enfermedad cardiovascular establecida, enfermedad renal, diabetes, riesgo cardiovascular a 10 años equivalente al $20 \%$ o superior para las metas de paciente de alto riesgo cardiovascular (Ib-Altamente recomendado) (recomendación adecuada o de primera línea) $)^{5,33,34}$

- Iniciar tratamiento con medicamentos antihipertensivos a personas de cualquier edad con hipertensión en etapa 2 (Ib-Altamente recomendado) (recomendación adecuada o de primera línea) $)^{5,33,34}$.

- Tanto como sea posible, recomiende el tratamiento con medicamentos tomados solo una vez al día, en única dosis o combinaciones en un solo comprimido (lb-Altamente recomendado) (recomendación adecuada o de primera línea) $)^{5,33,34}$

- Los pacientes con hipertensión arterial sistólica aislada con cifras iguales o mayores de 160 deberán recibir manejo. Ofrezca a las personas con hipertensión sistólica aislada (presión arterial sistólica de 160 o más), el mismo tratamiento que las personas con presión arterial sistólica y diastólica elevadas (Ib-moderadamente recomendado) (recomendación adecuada o de primera línea) 5,33,34.

- Ofrezca a personas de 70 años o más, el mismo tratamiento con medicamentos antihipertensivos que a las personas de entre 55 y 70 años, teniendo en cuenta las comorbilidades (Ib-moderadamente recomendado) (recomendación adecuada o de primera línea) ${ }^{5,33,34}$.

- Existe evidencia de para iniciar tratamiento antihipertensivo con un bloqueador de canales de calcio (BCC) en personas mayores de 60 años y en personas de raza negra de cualquier edad. Si no es adecuado un inhibidor de canal de calcio (CCB), ofrezca un diurético similar a la tiazida (lb-Altamente recomendado) (recomendación adecuada o de primera línea) ${ }^{5,33,34}$.

- En pacientes de raza negra los diuréticos (incluyendo espironolactona) en compañía de los CCB, son los medicamentos de primera línea de tratamiento (Ib-Altamente recomendado) (recomendación adecuada o de primera línea) ${ }^{5,33,34}$.

- Los betabloquedores no son una terapia inicial para la hipertensión. Sin embargo, los betabloqueadores se pueden considerar en personas más jóvenes, particularmente en:

- Aquellos con intolerancia o contraindicación a los IECA y ARA II o mujeres en edad fértil o personas con evidencia de mayor impulso simpático (lb-Altamente recomendado) (recomendación adecuada o de primera línea) $)^{5,33,34}$.

- Si la terapia se inicia con un betabloqueador y se necesita un segundo fármaco, agregue un bloqueador de los canales de calcio dihidropiridínico, en lugar de un diurético similar a la tiazida para reducir el riesgo de desarrollar hiperglicemia (lb-Altamente recomendado) (recomendación adecuada o de primera línea $)^{5,33,34}$.

- Se debe iniciar terapia combinada como primera línea de manejo en todo paciente con hipertensión arterial estadio 
Il (160/100) al momento del diagnóstico inicial, especialmente si es un paciente de alto riesgo cardiovascular (lb-Altamente recomendado) (recomendación adecuada o de primera línea) $)^{5,33,34}$.

- Se debe ofrecer un tratamiento escalonado teniendo en consideración los grupos farmacéuticos mencionados anteriormente, que incluyen CCB - IECA - ARB - diuréticos, bloqueadores alfa (Ib-Altamente recomendado) (recomendación adecuada o de primera línea) ${ }^{5,33,34}$.

\section{Pregunta 6 ¿A qué tipo de pacientes se le debe suministrar terapia combinada? ¿Qué medicamentos se deben usar en combinación?}

- La terapia combinada para el tratamiento de hipertensión arterial se refiere al uso de dos o más fármacos con diferente mecanismo de acción que tienen como objetivo controlar las cifras de tensión arterial a las metas requeridas y mantenerlas en el tiempo, aprovechando sus efectos sinérgicos y reduciendo los efectos adversos derivados del uso de altas dosis de los medicamentos por separado (lbAltamente recomendado) (recomendación adecuada o de primera línea) ${ }^{35}$.

- Los principales motivos para utilizar terapia combinada en pacientes hipertensos son los siguientes (la-Altamente recomendado) (recomendación adecuada o de primera línea) $)^{35}$ :

A) La alta frecuencia de pacientes con tensión arterial no controlada a pesar del tratamiento inicial, la cual se ha reportado entre el $30-54 \%{ }^{36}$.

B) Es el tratamiento recomendado de primera línea en pacientes con alto riesgo cardiovascular con daño subclínico de órgano (diabetes, enfermedad renal crónica, enfermedad cardiovascular) ${ }^{5}$.

C) Cuando la monoterapia a dosis máxima falla en controlar las tensiones arteriales.

D) La lógica del uso de la terapia combinada está basada en que la formulación de un único grupo antihipertensivo lleva a la activación de vías alternas fisiopatológicas que perpetúan la hipertensión (ejemplo: al usar un diurético se activa el eje renina-angiotensina-aldosterona). Por tanto, emplear dos o más fármacos de mecanismo de acción diferentes puede potenciar el efecto antihipertensivo de cada uno de ellos.

- Los beneficios de utilizar terapia combinada en hipertensión incluyen el mayor efecto hipotensor comparado con su uso por separado (efecto sinérgico), mejora la adherencia al tratamiento y podría optimizar el perfil de efectos adversos de las dosis máximas de fármacos en monoterapia. Más aún, la terapia combinada toma importancia dada la evolución reciente de las metas de tensión arterial que sugieren un manejo más intensivo de la hipertensión para obtener cifras que disminuyen el riesgo cardiovascular y mortalidad ${ }^{37,38}$ (la- Altamente recomendado) (recomendación adecuada o de primera línea).

- La recomendación actual para iniciar tratamiento combinado con dos fármacos de diferente mecanismo de acción como primera línea en hipertensión arterial involucra pacientes con diagnóstico confirmado de hipertensión arterial estadio 2 (guías previas con punto de corte de PAS/PAD $\geq 160 / 100 \mathrm{~mm} \mathrm{Hg;} \mathrm{guías} 2017$ americanas dan punto de corte de $\geq 140 / 90 \mathrm{~mm} \mathrm{Hg}$ ) con tensiones en promedio de $20 / 10 \mathrm{~mm} \mathrm{Hg}$ arriba de la meta deseada que significa más o menos arriba de $150 / 90 \mathrm{~mm} \mathrm{Hg}^{5}$ (la-Altamente recomendado) (recomendación adecuada o de primera línea).

- Pensar en terapia combinada obliga al médico a evaluar los beneficios que desea brindarle al paciente al emplear dicha combinación antihipertensiva; por consiguiente, se debe tener en cuenta el perfil de riesgo del paciente, las comorbilidades y los potenciales efectos benéficos a nivel de órganos entre los que se incluyen protección renal y protección cerebrovascular, metabólica $\mathrm{y} / \mathrm{o}$ cardiovascular ${ }^{37}$ (la-Altamente recomendado) (recomendación adecuada o de primera línea).

- Para realizar una terapia combinada exitosa se dispone de cuatro grupos farmacológicos principales: IECA, ARA, CCB y diuréticos (la-Altamente recomendado) (recomendación adecuada o de primera línea) $)^{5,39}$.

\section{Combinación de dos medicamentos}

- Algunas combinaciones NUNCA deben utilizarse ya que potencializan los efectos adversos de los medicamentos. La combinación que clásicamente debe evitarse es aquella en la que se utilicen dos o más medicamentos que inhiban el sistema renina-angiotensina (ej.: IECA + ARA, IECA y/o ARA + inhibidor de la renina) (la-Altamente recomendado) (recomendación adecuada o de primera línea).

Otras combinaciones que deben evitarse se detallan en la tabla 1.

- Las combinaciones más recomendadas por guías que aplican para Colombia son (lb- Altamente recomendado) (recomendación adecuada o de primera línea) ${ }^{5,40,41}$ :

- IECA con diuréticos: Enalapril+clortalidona/indapamida/ hidroclorotiazida (HCTZ).

Captopril + clortalidona/indapamida/hidroclorotiazida. Perindopril/indapamida

\section{- IECA con CCB:}

Enalapril + amlodipino.

Enalapril + nifedipino.

- ARA con diuréticos:

Losartan+clortalidona/indapamida/hidroclorotiazida. Valsartan + clortalidona/indapamida/hidroclorotiazida. Irbesartan + clortalidona/indapamida/hidroclorotiazida.

\section{- ARA con CCB:}

Losartan + amlodipino.

Valsartan + amlodipino. telmisartan + amlodipino. 
Tabla 1 Combinaciones no deseadas cuando se usan varios antihipertensivos y sus efectos adversos potenciales

\begin{tabular}{ll}
\hline Combinaciones que deben evitarse & Efectos adversos potenciales \\
\hline Betabloqueadores y clonidina & Bloqueo AV avanzado, disfunción del nodo sinusal. \\
Betabloqueadores y calcioantagonistas no dihidropiridínicos & Falla cardiaca y bradiarritmias \\
Bloqueadores alfa-1 y clonidina & Hipotensión sintomática \\
IECA +ARA & Disfunción renal \\
IECA o ARA + inhibidores de la renina (o la combinación de los tres) & Disfunción renal, enfermedad cerebrovascular isquémica.
\end{tabular}

Fuente: elaboración propia de los autores

- CCB con diuréticos:

Amlodipino + clortalidona/indapamida/hidroclorotiazida. Nifedipino + clortalidona/indapamida/hidroclorotiazida. Teniendo en cuenta las combinaciones anteriores que pueden realizarse en pacientes colombianos, a continuación, se resume la principal evidencia disponible obtenida de los grandes ensayos clínicos y sus beneficios cardiovasculares.

- Las combinaciones basadas en diltiazem tampoco han mostrado ser mejores que los tratamientos combinados basados en betabloqueadores ${ }^{42}$. Se puede concluir, gracias a lo anterior, que al escoger una terapia combinada de IECA + calcioantagonista se debería preferir el uso de calcioantagonistas dihidropiridínicos (amlodipino, nifedipino) que ha demostrado disminuir eventos cardiovasculares mayores ${ }^{43}$ (Ila-recomendado) (recomendación adecuada o de primera línea).

- No se recomiendan las combinaciones con betabloqueadores considerando que un reciente metaanálisis ${ }^{44}$ desestimó el uso de estos para el manejo de la hipertensión arterial exponiendo que este grupo era muy inferior a la hidroclorotiazida a bajas dosis en cuanto a desenlaces cardiovasculares, y que tienen pobre control de la tensión arterial ${ }^{45}$.

- Se debe iniciar terapia combinada como primera línea de manejo en todo paciente con hipertensión arterial estadio II (160/100) al momento del diagnóstico inicial, especialmente si es un paciente de alto riesgo cardiovascular (llarecomendado) (recomendación adecuada o de primera línea) ${ }^{43}$ :

- Quienes más se benefician son aquellos que han presentado ya un primer evento cardiovascular mayor (infarto de miocardio, ataque cerebrovascular, estenosis carotídea y/o enfermedad arterial periférica) (lla-recomendado) (recomendación adecuada o de primera línea) ${ }^{43}$.

- Se recomienda usar terapia combinada en pacientes que utilizaron monoterapia inicialmente y fallaron en lograr el control de sus cifras de presión arterial a las metas que se exigen, es decir, persisten con tensiones mayores a $140 / 90 \mathrm{~mm} \mathrm{Hg}$ en al menos dos tomas hechas en el consultorio o mayores a $135 / 85 \mathrm{~mm} \mathrm{Hg}$ en el MAPA o AMPA. Estas metas son diferentes en pacientes con alto riesgo cardiovascular en quienes el punto de corte de presión arterial de control es menor y depende de su comorbilidad principal (Ila-recomendado) (recomendación adecuada o de primera línea $)^{43}$.

- Para Colombia, se considera que las combinaciones que vienen en tableta diaria única comparado con los medicamentos por separado en sus respectivas dosis, son igualmente beneficiosas en cuanto al impacto en eventos cardiovasculares; sin embargo, se hace énfasis en que el uso de dosis única diaria aumentaría la adherencia al tratamiento antihipertensivo la cual es baja en Colombia, a un nivel muy similar al reportado en el resto del mundo (lla-recomendado) (recomendación adecuada o de primera línea ${ }^{44}$. Las principales recomendaciones de evidencia de las combinaciones hipertensivas se presentan en la tabla 2.

\section{Combinación de tres medicamentos}

- Si el paciente con hipertensión requiere un tercer medicamento para controlar su presión arterial, las combinaciones más recomendadas son (lla- recomendado) (recomendación adecuada o de primera línea) ${ }^{44}$ :

- ARA + diurético + CCB:

Losartan + clortalidona/indapamida/hidroclorotiazida + amlodipino. Losartan + clortalidona/indapamida/ hidroclorotiazida + nifedipino. Valsartan + clortalidona/indapamida/hidroclorotiazida + amlodipino. Olmesartan + clortalidona/indapamida/hidroclorotiazida + amlodipino.

- IECA + diurético + CCB:

Enalapril + clortalidona/indapamida/hidroclorotiazida + amlodipino. Enalapril + clortalidona/indapamida/ hidroclorotiazida + nifedipino. Perindopril + indapamida + amlodipino.

Pregunta 7 ¿Cuál debe ser el esquema básico de tratamiento aplicable a los pacientes adultos con hipertensión en Colombia, según nivel de riesgo y en enfermedades preexistentes (enfermedad coronaria, insuficiencia cardíaca congestiva, fibrilación auricular, enfermedad cerebrovascular, insuficiencia renal crónica, diabetes mellitus, cirrosis, obesidad, trasplante)?

\section{Enfermedad coronaria}

- Preferiblemente se propone lograr el control adecuado de cifras tensionales sistólicas y diastólicas, con una meta de presión arterial de $130 / 80 \mathrm{~mm} \mathrm{Hg}$ principalmente con tratamiento con IECA. Esta puede variar según edad y fragilidad del paciente; por tanto, se hace énfasis en 
Tabla 2 Resumen de evidencia de las combinaciones antihipertensivas

\begin{tabular}{|c|c|c|c|c|c|c|}
\hline Tipo de combinación & Grupo activo & Comparador & $\begin{array}{l}\text { Número de } \\
\text { pacientes }\end{array}$ & Tipo de pacientes & $\begin{array}{l}\text { Disminución de PA en } \\
\mathrm{mm} \mathrm{Hg} \\
\text { (sistólica/diastólica }\end{array}$ & Desenlaces \\
\hline \multicolumn{7}{|l|}{ IECA O ARA + diurético } \\
\hline ADVANCE (11) & $\begin{array}{l}\text { Perindopril } \\
\text { /Indapamida }\end{array}$ & Placebo & 11140 & Diabéticos & $-5.6 / 2.2 \mathrm{~mm} \mathrm{Hg}^{*}$ & $\begin{array}{l}\text { RRR: } 9 \% \text { eventos } \\
\text { macro/micro } \\
\text { vasculares } \\
\text { RRR: } 18 \% \text { muerte } \mathrm{CV}^{*}\end{array}$ \\
\hline PROGRESS (12) & Perindopril /Indapamida & Perindopril /placebo & 6105 & ACV & $-9 / 4 \mathrm{~mm} \mathrm{Hg}^{*}$ & RRR: $28 \%$ de $A C V *$ \\
\hline HYVET (13) & $\begin{array}{l}\text { Indapamida/placebo } \\
\text { Perindopril si necesario }\end{array}$ & placebo & 3845 & HSA >80 años & $-14.5 / 6.8 \mathrm{~mm} \mathrm{Hg}^{*}$ & RRR: $34 \%$ eventos CV \\
\hline CAPP (14) & Captopril/D & $\mathrm{BB} / \mathrm{D}$ & 10985 & Hipertensos & $+3 \mathrm{~mm} \mathrm{Hg}^{+}$ & Mas $5 \%$ de eventos ${ }^{+}$ \\
\hline \multicolumn{7}{|c|}{ IECA + Calcio antagonista } \\
\hline ACCOMPLISH (15) & Benazepril/Amlodipino & $\begin{array}{l}\text { Benazepril/ } \\
\text { Hidroclorotiazida }\end{array}$ & 11506 & $\begin{array}{l}\text { Hipertensos } \\
\text { Mas factores de riesgo }\end{array}$ & SD & RRR: $19.6 \%$; RRA $2.2 \%$ \\
\hline ASCOT (16) & Perindopril/amlodipino & $\begin{array}{l}\text { Atenolol/ } \\
\text { bendroflumetiazida }\end{array}$ & 19257 & $\begin{array}{l}\text { Hipertensos } \\
\text { Mas factores de riesgo }\end{array}$ & $-2.7 / 1.9 \mathrm{~mm} \mathrm{Hg}{ }^{*}$ & $\begin{array}{l}\text { RRR: } 23 \% \text { mortalidad } \\
\text { CV, } 16 \text { eventos CV }\end{array}$ \\
\hline INVEST (17) & Verapamilo/ trandolapril & $\begin{array}{l}\text { Atenolol/ } \\
\text { Hidroclorotiazida }\end{array}$ & 22576 & Hipertensos con EC & SD & SD en eventos CV \\
\hline NORDIL (18) & Diltiazem/IECA & $\mathrm{BB}$ y/o D/IECA & 10881 & Hipertensos & $\begin{array}{l}-3 \mathrm{mmHG} \text { /SD } \\
\text { diastólicas }\end{array}$ & SD en eventos CV \\
\hline \multicolumn{7}{|c|}{ Calcio antagonista + diurético } \\
\hline FEVER (20) & $\begin{array}{l}\text { Felodipino/ } \\
\text { Hidroclorotiazida }\end{array}$ & $\begin{array}{l}\text { Hidroclorotiazida/ } \\
\text { Placebo }\end{array}$ & 9711 & Hipertensos & $-4.2 / 2.1 \mathrm{~mm} \mathrm{Hg}$ & RRR: $35 \%$ Eventos CV. \\
\hline ELSA (21) & Lacidipina con o sin D & Atenolol con o sin D & 2334 & $\begin{array}{l}\text { Hipertensos enfermedad } \\
\text { carotidea }\end{array}$ & SD & SD en eventos CV \\
\hline CONVINCE (22) & Diltiazem/ BB & $\mathrm{BB} /$ placebo o D & 16602 & $\begin{array}{l}\text { Hipertensos de alto } \\
\text { riesgo }\end{array}$ & SD & SD en eventos CV \\
\hline VALUE & Amlodipino Con o sin D & ARA/ con o sin D & 13449 & $\begin{array}{l}\text { Hipertensos de alto } \\
\text { riesgo }\end{array}$ & $\begin{array}{l}-2.2 \mathrm{~mm} \mathrm{\textrm {Hg } ^ { + } / \mathrm { SD }} \\
\text { diastólicas }\end{array}$ & $\begin{array}{l}\text { RRR: } 3 \% \text { eventos CV } \\
\text { NS }^{+}\end{array}$ \\
\hline \multicolumn{7}{|l|}{$A R A+$ diurético } \\
\hline SCOPE (24) & $\begin{array}{l}\text { Candesartan/ } \\
\text { Hidroclorotiazida }\end{array}$ & Hidroclorotiazida/ & 4964 & Hipertensos $>70$ años & $-3.2 / 1.6 \mathrm{~mm} \mathrm{Hg}^{*}$ & $\begin{array}{l}\text { RRR: } 28 \% \text { ACV no fatal } \\
\text { ", sin otros desenlaces }\end{array}$ \\
\hline LIFE (25) & $\begin{array}{l}\text { Losartan/ } \\
\text { Hidroclorotiazida }\end{array}$ & $\begin{array}{l}\text { Placebo } \\
\text { Atenolol/ } \\
\text { Hidroclorotiazida }\end{array}$ & 9193 & Hipertensos con HVI & $\begin{array}{l}-1 \mathrm{~mm} \mathrm{Hg}^{+} / \mathrm{SD} \\
\text { diastólicas }\end{array}$ & RRR: $26 \% \mathrm{ACV}^{*}$ \\
\hline \multicolumn{7}{|c|}{$\begin{array}{l}\text { ECA: inhibidores de la enzima convertidora de angiotensina; ARA: Antagonistas del receptor de la angiotensina; RRR: Reducción del riesgo relativo, ACV: Accidente cerebro vascular; HSA: } \\
\text { Hipertensión sistólica asilada; CV: cardiovasculares; BB: betabloqueador; D: diurético; EC: enfermedad coronaria; SD: sin diferencias; NS: no significativo; HVI: Hipertrofia de ventrículo } \\
\text { izquierdo }\end{array}$} \\
\hline
\end{tabular}


pacientes mayores de 70 años (lla-recomendado) (recomendación adecuada o de primera línea $)^{4,44,45}$.

- Los betabloqueadores SON efectivos en la disminución de aparición de angina de pecho, pues mejoran la capacidad física y disminuyen la aparición de dolor torácico inducido por el ejercicio, así como los cambios del segmento ST desencadenado con actividad física, principalmente con carvedilol, metoprolol tartrato, metoprolol succinato, propanolol y bisoprolol, reduciendo la mortalidad en un $23 \%$. Es por esto que se indica para el paciente hipertenso (Ib-Altamente recomendado) (recomendación adecuada o de primera línea) $)^{4,46}$.

- Los calcioantagonistas dihidropiridínicos también son efectivos en adición a betabloqueadores, para la prevención de la angina con cifras tensionales menores de $120 \mathrm{~mm} \mathrm{Hg}$, cuando las cifras tensionales persisten elevadas pues se logra disminuir el riesgo de enfermedad cerebrovascular (lla- recomendado) (recomendación adecuada o de primera línea $)^{4,47}$.

- Los calcioantagonistas no dihidropiridínicos (diltiazem, verapamilo), se pueden usar en pacientes con angina estable si los betabloqueadores están contraindicados, pero no en de manera concomitante y si hay falla cardiaca asociada (lb-Altamente recomendado) (recomendación adecuada o de primera línea $)^{4,48}$.

- Los diuréticos tiazídicos (hidroclorotiazida, indapamida y clortalidona) han demostrado disminuir eventos cardiovasculares, idealmente clortalidona sobre hidroclorotiazida (Ila-recomendado) (recomendación adecuada o de primera línea $)^{4,49}$.

- En enfermedad coronaria hay un comportamiento en J, sobre todo en cifras diastólicas, siendo más favorable manejar cifras tensionales diastólicas entre 70 y $80 \mathrm{~mm}$ $\mathrm{Hg}$. Se observan mayores eventos con cifras tensionales diastólicas mayores de $80 \mathrm{~mm} \mathrm{Hg}$ y menores de $70 \mathrm{~mm}$ Hg (Registro CLARIFY) (Ila recomendada) (recomendación adecuada o de primera línea $)^{4,48,49}$.

- Las cifras tensionales diastólicas menores de $60 \mathrm{~mm} \mathrm{Hg}$ están asociadas a enfermedad coronaria y aumento de la mortalidad (Ila-recomendado) (recomendación adecuada o de primera línea $)^{4,48,49}$.

- La disminución de al menos $10 \mathrm{~mm}$ Hg en las cifras tensionales disminuía en aproximadamente el $20 \%$ de eventos cardiovasculares mayores, $17 \%$ en enfermedad coronaria, $27 \%$ en enfermedad cerebrovascular, $28 \%$ en falla cardiaca y $13 \%$ en mortalidad por todas las causas (Ilarecomendado) (recomendación adecuada o de primera línea $)^{4,48,49}$.

- En síndrome coronario agudo se pueden usar betabloqueadores endovenosos, preferiblemente esmolol, para el control de las cifras tensionales (lla-recomendado) (recomendación adecuada o de primera línea $)^{4,48,49}$.

- Los medicamentos nitratos se pueden utilizar excepto en aquellos pacientes infarto del ventrículo derecho (Ilarecomendado) (recomendación adecuada o de primera línea) $)^{4,48,49}$.

- En falla cardiaca, la hipertensión está presente en $75 \%$ de los pacientes. Estudios con betabloqueadores han demostrado mejoría en la sobrevida en estos pacientes con metoprolol succinato, bisoprolol, carvedilol, nebivolol y con antagonistas de la aldosterona (espironolactona y eplerenona) (Ila-recomendado) (recomendación adecuada o de primera línea) $)^{4,49,50}$.

- En pacientes con hipertensión la clortalidona mostró menor aparición de falla cardiaca con freccion de eyección reducida (Ila). Al igual que el lisinopril, ambos fueron superiores a amlodipino y daxozacina (Ila-recomendado) (recomendación adecuada o de primera línea) ${ }^{44,48,49}$.

- La espironolactona en falla cardiaca con fracción de eyección conservada disminuyó los desenlaces cardiovasculares, lo que podría ser útil para la población colombiana por las características demográficas de los pacientes y en el caso de tener indicación en pacientes con falla cardiaca y fracción de eyección conservada y persistencia de hipertensión arterial (Ila-recomendado) (recomendación adecuada o de primera línea) $)^{4,48,49}$.

\section{Fibrilación auricular}

- En estos pacientes el riesgo de eventos tromboembólicos es mayor con cifras tensionales no controladas, es decir, PAS mayor de $140 \mathrm{~mm} \mathrm{Hg}$ y PAD mayor de $90 \mathrm{~mm}$ $\mathrm{Hg}$, así como, riesgo alto de sangrado no mayor, clínicamente relevante. Se deben utilizar medicamentos de vida media prolongada para prevenir variabilidad de la presión arterial visita a visita, la cual cuando no está controlada, aumenta el riesgo de eventos cardiovasculares. La meta de presión arterial en estos pacientes son cifras menores de $140 / 90 \mathrm{~mm} \mathrm{Hg}$, en tanto que, en pacientes mayores de 80 años con fibrilación auricular, cifras menores de 150/90 mm Hg (III-recomendadas sólo si el criterio clínico ve utilidad $)^{4,48,49}$.

- Mantener actividad física regular es muy importante en los pacientes con fibrilación auricular pues disminuye la recurrencia de eventos con mejor control del ritmo, reduce la de fibrilación auricular y los eventos libres de hospitalización, y controla mejor las cifras tensionales (III-recomendadas solo si el criterio clínico ve utilidad) (recomendación adecuada o de primera línea) $)^{4,48,49}$.

\section{Diabetes mellitus}

- No se ha no demostrado mejoría en desenlaces cardiovasculares en pacientes con diabetes con el control de cifras tensionales de forma intensiva (PAS menor de $120 \mathrm{~mm}$ $\mathrm{Hg}$ ), en comparación con un grupo control (PAS menor de $140 \mathrm{~mm} \mathrm{Hg}$ ). Se debe asegurar que al tratar un hipertenso con diabetes asociada se incluya en el tratamiento al menos un IECA o ARA ya sea solo o en combinación con un calcioantagonista, con el fin de llegar a la meta de tensiones y a su vez reducir la aparición y deterioro de una nefropatía diabética. La meta en general es llevar a tensiones menores de $140 / 80 \mathrm{~mm} \mathrm{Hg}$ (III-recomendadas solo si el criterio clínico ve utilidad) (recomendación adecuada o de primera línea).

\section{Enfermedad renal crónica}

- El riesgo cardiovascular se eleva en pacientes con enfermedad renal crónica con cifras tensionales por encima de $115 / 75 \mathrm{~mm} \mathrm{Hg}$ y se duplica por cada elevación de $20 / 10 \mathrm{~mm} \mathrm{Hg}$, esto al evaluar enfermedad renal en 
pacientes no diabéticos (Illb-recomendado) (recomendación adecuada o de primera línea $)^{4,50,51}$.

- Se recomienda manejar cifras de tensión < 125-130/80 mm $\mathrm{Hg}$, en pacientes con enfermedad renal crónica no diabéticos para disminuir la tasa de eventos cardiovasculares mayores y todas las causas de mortalidad (recuerde que la proteinuria muestra el cumplimiento de metas). Cifras tensionales $<125-130 / 75-80 \mathrm{~mm}$ Hg no fueron más benéficas que cifras tensionales $<140 / 90 \mathrm{~mm} \mathrm{Hg}$ en cuanto a mejorar los resultados renales y cardiovasculares, pero deben ser consideradas en pacientes con proteinuria $>300-1.000 \mathrm{mg} / \mathrm{dl}$ con base en análisis de subgrupos (Ilarecomendada) (recomendación adecuada o de primera línea) $)^{4,50,51}$.

- En estos pacientes, el uso de antagonistas de la aldosterona (espironolactona), disminuyó la proteinuria en un $58 \%$, pero con mayor evidencia de hipercalemia, por lo cual se recomienda la monitorización continua de la función renal y el potasio en este grupo de pacientes (Ilarecomendado) (recomendación adecuada o de primera línea $)^{4,50,51}$.

- Los calcioantagonistas no dihidropiridínicos en combinación con IECA o ARA II, pueden ser usados como alternativa en proteinuria persistente y cifras tensionales persistentemente elevadas, aunque no se ha demostrado la preservación del a TFG en la práctica (III-recomendadas solo si el criterio clínico ve utilidad) ${ }^{4,50,51}$.

- No se recomienda el uso concomitante de IECA, ARA II o inhibidor directo de la renina por aumento del riesgo de hipercalemia y deterioro de la función renal en este grupo de pacientes (llb-recomendado) (recomendación adecuada o de primera línea $)^{4,50,51}$.

\section{Trasplante renal}

- Los objetivos del control de cifras tensionales cambian con el tiempo del trasplante; inicialmente se recomiendan cifras tensionales menores de $160 / 90 \mathrm{~mm} \mathrm{Hg}$ para mantener perfusión adecuada del órgano trasplantado, y disminuir el riesgo de hipotensión y trombosis del injerto. Luego de un mes de trasplante el objetivo es igual que para pacientes no trasplantados a fin de evitar daño de órgano blanco (lla-recomendado) (recomendación adecuada o de primera línea $)^{4,52,53}$.

\section{Enfermedad cerebrovascular}

- La razón para reducir la presión arterial en este caso es prevenir el edema cerebral y limitar la transformación hemorrágica del infarto. La preocupación por disminuir la presión arterial es la expansión del núcleo isquémico central al empeorar la hipoperfusión dentro de la penumbra isquémica, porque esta área puede haber interrumpido la autorregulación cerebral. Luego de un evento isquémico cerebral, el $75 \%$ de los pacientes tienen cifras tensionales superiores a $140 \mathrm{~mm} \mathrm{Hg}$ durante los primeros dos días del evento, y el $25 \%$ presentan cifras tensionales mayores de $180 \mathrm{~mm} \mathrm{Hg}$; por consiguiente, se recomienda reducir las cifras tensionales del 20 al 30\%, disminución que se asoció con adecuada evolución (Barcelona Downtown stroke registry) (Ib-Altamente recomendado) (recomendación adecuada o de primera línea $)^{4,54}$.
- Si un paciente es elegible para terapia trombolítica (activador del plasminógeno tisular), se recomienda mantener cifras tensionales menores de $185 / 110 \mathrm{~mm} \mathrm{Hg}$ y sostener este control durante las primeras 24 horas después de iniciada la terapia (lb-Altamente recomendado) (recomendación adecuada o de primera línea $)^{4,54}$.

- Pacientes con cifras tensionales menores de $155 \mathrm{~mm} \mathrm{Hg}$ tenían menos probabilidades de morir a 90 días que aquellos con cifras tensionales mayores (155 a $220 \mathrm{~mm} \mathrm{Hg}$ ), según el estudio CHHIPS, en el cual se realizó reducción intensiva de cifras tensionales en evento agudo, sin cambios significativos en desenlaces a corto plazo, pero con descenso de 20,3 a 9,7\% de la mortalidad a los tres meses así como de las cifras tensionales de $21 \mathrm{~mm} \mathrm{Hg}$ en el grupo de manejo activo (llb-recomendado) (recomendación adecuada o de primera línea $)^{4,54}$.

- Aunque la evidencia no es muy contundente las guías recomiendan el uso de nicardipina o labetalol para disminuir cifras tensionales. El reinicio de medicación antihipertensiva oral debe estar relacionado con la deglución adecuada para prevenir riesgo de broncoaspiración, lo cual aumenta la posibilidad de complicaciones (IIIrecomendadas solo si el criterio clínico ve utilidad) (no se recomienda) ${ }^{4,54}$.

- En pacientes con hemorragia intracerebral y cifras tensionales elevadas es importante mantener cifras de presión por encima de $140 \mathrm{~mm} \mathrm{Hg}$ para lograr una perfusión cerebral adecuada; las cifras tensionales menores de $100 \mathrm{~mm}$ $\mathrm{Hg}$ se asociaron con peor pronóstico y deterioro neurológico. No hubo cambios significativos en cifras tensionales por debajo de $150 \mathrm{~mm} \mathrm{Hg}$ y $180 \mathrm{~mm} \mathrm{Hg}$ (Ilb-recomendado) (recomendación adecuada o de primera línea) ${ }^{4,54}$.

\section{Prevención secundaria de eventos isquémicos posterior a evento (AIT)}

- Se recomienda iniciar terapia antihipertensiva a los pocos días del evento para prevenir el riesgo de accidente isquémico recurrente $u$ otro evento vascular. La combinación de un diurético tiazídico mas IECA o ARA II es la que tiene mejor evidencia para disminuir el riesgo de nuevo accidente isquémico cerebral (Ila-recomendado) (recomendación adecuada o de primera línea) ${ }^{4}$.

- Pacientes con hipertensión arterial y enfermedad arterial periférica deben ser tratados de forma similar que pacientes hipertensos sin enfermedad arterial periférica. Se recomienda el uso de IECA y ARA II para controlar el riesgo cardiovascular (lla- recomendado) (recomendación adecuada o de primera línea $)^{4}$.

- Los betabloqueadores no empeoran la claudicación intermitente, incluso el nebivolol parece mejorar un poco la posibilidad de claudicación en algunos pacientes; en estenosis carotídea se recomiendan los antagonistas de los canales de calcio y los IECA (Ilb-recomendado) (recomendación adecuada o de primera línea $)^{4}$.

\section{Síndrome metabólico}

- No hay claridad sobre el manejo de la hipertensión arterial en este caso, por tanto, se recomiendan terapias no farmacológicas que mejoren los hábitos alimentarios (llbrecomendado) (recomendación adecuada o de primera línea $)^{4}$. 
- Los diuréticos tiazídicos se prefieren evitar por la alteración metabólica que producen, aumentando el riesgo de diabetes e hiperuricemia que puede acompañar esta alteración (lla-recomendado) (recomendación adecuada o de primera línea).

- Los betabloqueadores en general produjeron alteración metabólica en el 15 al 29\%, sin embargo, los betabloqueadores vasodilatadores (labetalol, carvedilol, nebivolol), se mostraron metabólicamente neutros (IIb- recomendado) (recomendación adecuada o de primera línea).

\section{Obesidad}

- En pacientes obesos la recomendación es similar que, para pacientes no obesos, aunque se refuerzan las medidas no farmacológicas relacionadas con la pérdida de peso y la actividad física regular (Ilb-recomendado) (recomendación adecuada o de primera línea).

- Por cada pérdida de $1 \mathrm{~kg}$ de peso, se disminuye 1,05/0,92 mm Hg de cifras de presión arterial (recomendación adecuada o de primera línea $)^{5}$.

- El orlistat inhibe la lipasa intestinal y su uso se asocia con disminución de $2,7 \mathrm{~kg}$ de peso teniendo bajo efecto sobre las cifras de presión arterial (lb- Altamente recomendado) (recomendación adecuada o de primera línea).

- El liraglutide (agonista del GLP-1) también se ha asociado con disminución de peso en pacientes diabéticos, con pérdida de hasta $7,8 \mathrm{~kg}$ de peso acompañada de disminución de $12,5 \mathrm{~mm} \mathrm{Hg}$ de las cifras de presión arterial, aunque aumenta $3 \mathrm{lpm}$ la frecuencia cardiaca en promedio (IbAltamente recomendado) (recomendación adecuada o de primera línea) $)^{4}$.

- Como última opción está la cirugía bariátrica en pacientes con obesidad extrema que requieren bajar de peso (IbAltamente recomendado) (recomendación adecuada o de primera línea) $)^{4}$.

\section{Cirrosis hepática}

- Se recomienda el uso de bloqueadores de los canales de calcio, alfabloqueadores y diuréticos de ASA, betabloqueadores y diuréticos ahorradores de potasio (IbAltamente recomendado) (recomendación adecuada o de primera línea) $)^{4,55,56}$

- La utilización de IECA y ARA II, se limitó en pacientes con cirrosis por el potencial riesgo de nefropatía (lbAltamente recomendado) (recomendación adecuada o de primera línea $)^{4,55,56}$.

\section{Ascitis severa}

- Se recomienda el uso de propranolol en lugar de carvedilol dado el riesgo de hipotensión con este último (III-recomendado solo si el criterio clínico ve utilidad $)^{4}$.

\section{Pregunta 8 ¿Cuáles son las indicaciones de uso del ecocardiograma en el seguimiento del paciente adulto con hipertensión arterial?}

- Se recomienda la ecocardiografía transtorácica para la evaluación del riesgo cardiovascular en pacientes asintomáticos adultos con hipertensión y la evaluación de su repercusión estructural cardiaca (Ilb-Recomendado) (recomendación adecuada o de primera línea $)^{57-61}$.

- El ecocardiograma de estrés con ejercicio o estímulo farmacológico está indicado en hipertensos con síntomas que sugieran enfermedad coronaria y para valorar el pronóstico en pacientes con enfermedad coronaria concomitante conocida, así como aquellos con enfermedad cardíaca valvular conocida o sospechada (Ilbrecomendado) (recomendación adecuada o de primera línea) $)^{57-61}$.

- En la actualidad, las decisiones respecto al inicio, la intensificación o el control de la respuesta a las terapias antihipertensivas se basan en parámetros clínicos y no en el ecocardiograma (lb-Altamente recomendado) (recomendación adecuada o de primera línea $)^{57-61}$.

- Dada la naturaleza progresiva de la miocardiopatía hipertensiva, la evaluación periódica de la función cardíaca y la morfología por ecocardiografía pueden estar justificadas, especialmente si los síntomas cambian o hay indicios de hipertrofia ventricular izquierda (Ila-recomendado) (recomendación adecuada o de primera línea $)^{57-61}$.

- En pacientes con diagnóstico reciente de hipertensión se realizará ecocardiogramna y el seguimiento con este método dependerá del criterio clínico (Ila-recomendado) (recomendación adecuada o de primera línea) ${ }^{57-61}$.

\section{Pregunta 9 ¿Respecto a la medicina alternativa y las terapias complementarias, ¿qué evidencia tienen en el manejo de la hipertensión arterial?}

- Se recomienda no suspender terapias alternativas como coadyuvantes del tratamiento farmacológico y no farmacológico instaurado en un paciente con diagnóstico de hipertensión arterial (fitoterapia, acupuntura, yoga, qigong, taichí, meditación) (Ila-recomendado) (recomendación adecuada o de primera línea) $)^{70-77}$.

- Solo los expertos en terapias alternativas de programas universitarios avalados y con nivel de maestría podrán formular estas terapias a los pacientes con diagnóstico de hipertensión arterial (lla-recomendado) (recomendación adecuada o de primera línea).

- No hay evidencia clínica de estudios occidentales que respalden el manejo de la hipertensión arterial con terapias alternativas.

\section{Pregunta 10 ¿Cuál debe ser el manejo de la hipertensión arterial en edades especiales (niños, jóvenes y ancianos)?}

- Es importante aclarar que, al tratarse de un tópico pediátrico, que escapa al objetivo general de este consenso, se sugiere que, para mayor estudio y profundización, se revise la bibliografía anexa, en especial la guía de práctica clínica para el diagnóstico y manejo de la hipertensión arterial en niños y adolescentes publicada por la American Academy of Pediatrics en el año $2017^{78}$.

- En niños es importante tener en cuenta el cambio que se realizó a la definición de estadios de la presión arterial, los cuales se observan en la tabla 3 . 
Tabla 3 Definición de los estadios de presión arterial en niños

\begin{tabular}{|c|c|c|}
\hline & Niños entre 1 a 13 años & Niños mayores de 13 años \\
\hline PA normal & $<\mathrm{PC} 90$ & $<120 /<80$ \\
\hline PA elevada & $\begin{array}{l}>\text { PC } 90-95, \text { o, > 120/80 a < PC } 95 \text { (el que se más } \\
\text { bajo) }\end{array}$ & $120 /<80$ a $129 /<80$ \\
\hline hipertensión arterial estadio 1 & $\begin{array}{l}>\text { PC } 95 \text { a < PC } 95+12 \mathrm{~mm} \mathrm{Hg}, 0,130 / 80-139 / 89 \text { (el } \\
\text { que se más bajo) }\end{array}$ & $130 / 80$ a $139 / 89$ \\
\hline $\begin{array}{l}\text { hipertensión arterial estadio } 2 \\
\text { PA: presión arterial, } P C \text { : percentil, }\end{array}$ & > PC $95+12 \mathrm{~mm} \mathrm{Hg}$, o, > 140/90 (el que se más bajo) & $>140 / 90$ \\
\hline
\end{tabular}

La evaluación inicial en todos los pacientes con hipertensión arterial incluye: parcial de orina, gram de orina, BUN, creatinina, electrolitos y perfil lipídico completo. En niños o adolescentes con índice de masa corporal mayor al percentil 95 se debe ordenar, además, hemoglobina A1c y transaminasas. Algunos test adicionales que se deben realizar en busca de causas secundarias incluyen: hormonas tiroideas (hipertiroidismo), glucosa e insulina en ayunas (diabetes), resonancia magnética nuclear o angiografía renal (sospecha de estenosis de la arteria renal o malformaciones renales), niveles séricos de esteroides y catecolaminas (sospecha de feocromocitoma), polisomnografía del sueño (sospecha de apnea del sueño), hemograma (pacientes con función renal anormal o retardo en el crecimiento), tamizaje de sustancias psicoactivas (la-Altamente recomendado) (recomendación adecuada o de primera línea) $)^{78-80}$.

- En pacientes jóvenes o en aquellos con hipertensión arterial estadio 2, se debe evaluar la medición de la actividad de la renina plasmática y niveles de aldosterona al momento del diagnóstico (la-Altamente recomendado) (recomendación adecuada o de primera línea) ${ }^{79-82}$.

- Además de intentar determinar la presencia de etiologías secundarias, los niños y adolescentes con presión arterial elevada requieren una evaluación exhaustiva para determinar daño de órganos diana y riesgo cardiovascular. Esta evaluación debe incluir (la-Altamente recomendado) (recomendación adecuada o de primera línea) $)^{79-82}$.

- Ecocardiograma pediátrico: evalúa masa, geometría y función del ventrículo izquierdo, buscando determinar la presencia de hipertrofia ventricular izquierda.

- Ecografía renal Doppler: se usa como un estudio de tamizaje no invasivo para la detección de estenosis de la arteria renal en niños con peso normal y adolescentes $\geq 8$ años de edad con sospecha de hipertensión arterial renovascular.

- La evaluación clínica neurológica y oftalmológica está indicada en la hipertensión grave $\mathrm{g}^{79-82}$.

o En pacientes con presión arterial elevada o hipertensos, antes de iniciar la terapia médica, se recomiendan modificaciones en el estilo de vida, que incluyen (la-Altamente recomendado) (recomendación adecuada o de primera línea):

- Iniciar la dieta tipo DASH (The Dietary Approaches to Stop Hypertension), la cual ofrece una ingesta rica en legumbres, frutas, vegetales, lácteos bajos en grasas, granos integrales, carnes rojas magras, pescado, pollo, nueces. Además, es preciso limitar la ingesta de sodio, cafeína y azúcares procesados. Las calorías de la dieta deben repartirse de la siguiente manera: $25-30 \%$ provenientes de proteínas, $40-60 \%$ provenientes de carbohidratos y solo $20-30 \%$ provenientes de lípidos $^{18}$ (la-Altamente recomendado) (recomendación adecuada o de primera línea $)^{79,81,83}$.

- Recomendar la práctica de actividad física moderada a vigorosa, mínimo tres a cinco días a la semana, con una duración de treinta a sesenta minutos cada sesión. Cualquier tipo de ejercicio (aeróbico, de resistencia o combinado), parece ser beneficioso (la-Altamente recomendado) (recomendación adecuada o de primera línea $)^{79,84,85}$.

- Se ha encontrado que la pérdida de peso en pacientes con sobrepeso se asocia con disminución de la presión arterial; además, disminuye la sensibilidad de la presión arterial a la sal y otros factores de riesgo cardiovascular (la-Altamente recomendado) (recomendación adecuada o de primera línea) ${ }^{84,86}$.

- En algunos estudios, intervenciones de medicina no tradicional han demostrado ser prometedoras en niños y adolescentes normotensos y en aquellos con presión arterial elevada. La meditación con respiración consciente condujo a una reducción de la PAS $(3-4 \mathrm{~mm} \mathrm{Hg})$ y de la PAD $(1 \mathrm{~mm} \mathrm{Hg})$ durante las 24 horas en adolescentes afroamericanos normotensos y con presión arterial elevada (Ilb-recomendado) (recomendación adecuada o de primera línea).

- Evitar el sedentarismo, disminuir el uso de dispositivos electrónicos como celular, tablet, computador, a máximo dos horas/día (la-Altamente recomendado) (recomendación adecuada o de primera línea $)^{79}$.

- $\mathrm{Si}$ el paciente no requiere manejo antihipertensivo (antiHTA) y se ha tomado la decisión de proceder solo con cambios en el estilo de vida, las citas de control se pueden realizar cada 3-6 meses para reforzar el cumplimiento de estos cambios y estar evaluando la necesidad de 
iniciar antiHTA (la-Altamente recomendado) (recomendación adecuada o de primera línea $)^{79}$.

\section{Tratamiento farmacológico}

Se indica manejo antihipertensivo a los pacientes que (laAltamente recomendado) (recomendación adecuada o de primera línea) ${ }^{79,84,87}$ :

- Permanecen hipertensos a pesar de modificaciones del estilo de vida

- Tienen hipertensión arterial sintomática

- Tienen hipertensión arterial con hipertrofia del ventrículo izquierdo en el ecocardiograma

- Tienen hipertensión arterial estadio 2 sin un factor claramente modificable (por ejemplo, obesidad)

- Tienen cualquier estadio de hipertensión arterial asociado con enfermedad renal crónica o diabetes mellitus.

- Por la alta prevalencia de hipertensión arterial secundaria, el tratamiento farmacológico debe ir encaminado al control de la etiología específica y el inicio de antiHTA que no estén contraindicados (la-Altamente recomendado) (recomendación adecuada o de primera línea) ${ }^{79}$.

- El médico tratante debe iniciar uno de los siguientes antiHTA (la-Altamente recomendado) (recomendación adecuada o de primera línea $)^{79}$ :

- IECA.

- ARAII.

- BCC de acción prolongada o un diurético tiazídico, siempre y cuando no exista contraindicación para su uso.

- Se debe iniciar un antiHTA en el rango inferior de dosificación y según las mediciones posteriores de la presión arterial, la dosis se puede aumentar cada 2 a 4 semanas hasta que se controle la presión arterial (< percentil 90), se alcance la dosis máxima o se produzcan efectos adversos (la-Altamente recomendado) (recomendación adecuada o de primera línea) $)^{79,87}$.

- El tratamiento de un niño o adolescente con hipertensión arterial requiere una monitorización continua porque la presión arterial objetivo puede ser difícil de lograr. El paciente debe ser controlado cada cuatro a seis semanas para realizar ajustes de dosis y/o adición de un segundo o tercer agente, hasta que se alcance la presión arterial objetivo. Una vez esta se haya normalizado las citas de control se pueden programar cada tres a cuatro meses. Si no se controla con un sólo medicamento, se puede agregar uno más y titularlo de la misma manera como se realizó con el antiHTA inicial. Debido a la retención de sal y agua que se produce con muchos antiHTA, un diurético tiazídico suele ser el segundo medicamento de preferencia (la-Altamente recomendado) (recomendación adecuada o de primera línea) ${ }^{79,87}$.

- En pacientes tratados con medicamentos antiHTA, se recomienda hacer seguimiento de la presión arterial con MAPA, especialmente en aquellos con enfermedad renal crónica (la-Altamente recomendado) (recomendación adecuada o de primera línea) ${ }^{79,87}$.
- Los betabloqueadores no se recomiendan como tratamiento inicial en niños, dado el perfil de efectos adversos y porque en adultos no han mostrado mejores resultados para el control de la hipertensión arterial cuando se comparan con otros antiHTA (la-Altamente recomendado) (recomendación adecuada o de primera línea $)^{79,87,88}$.

- En niños con hipertensión arterial y enfermedad renal crónica y/o proteinuria, el agente antiHTA inicial que se recomienda es un IECA o un ARAll, a menos que exista una contraindicación absoluta. En algunos casos estos medicamentos no son suficientes para controlar la presión arterial, de modo que se puede adicionar un diurético o un bloqueador de los canales de calcio (a veces se adiciona betabloqueador solo si la presión arterial objetivo no se logra combinando tres fármacos) (la-Altamente recomendado) (recomendación adecuada o de primera línea $)^{79,87,88}$.

- En niños con coartación de la aorta se recomienda iniciar betabloqueadores antes de la corrección quirúrgica, ya que reducen el riesgo de crisis hipertensivas posoperatorias. En algunos pacientes se mantiene el medicamento, ya que posterior a la intervención pueden desarrollar hipertensión arterial persistente. En ocasiones también pueden requerir IECA (la-Altamente recomendado) (recomendación adecuada o de primera línea) $)^{79,87}$.

- En niños con hipertensión arterial y diabetes mellitus, el agente antihipertensivo inicial que se recomienda es un IECA o un ARAll, a menos que exista una contraindicación absoluta. También pueden ser útiles los bloqueadores de canales de calcio ya que mejoran la sensibilidad a la insulina. Estos pacientes no deben usar betabloqueadores ya que se han asociado a resistencia a la insulina y además pueden enmascarar síntomas de hipoglicemia (laAltamente recomendado) (recomendación adecuada o de primera línea) $)^{79,87,89,90}$.

- Los pacientes con hipertensión arterial tienen mayor riesgo de dislipidemia ya que las dos enfermedades tienen origen común (obesidad, sedentarismo y mala alimentación) ${ }^{91}$. Por tanto, se recomienda el tratamiento de la dislipidemia según las guías pediátricas existentes (la-Altamente recomendado) (recomendación adecuada o de primera línea $)^{79,92}$.

- En pacientes con hipertensión arterial y migraña se deben usar betabloqueadores o bloqueador de los canales de calcio (la-Altamente recomendado) (recomendación adecuada o de primera línea $)^{82}$.

- Los bloqueadores de los canales de calcio pueden usarse en niños con falla renal, ya que, al tener metabolismo hepático, no tienen efectos renales similares a los IECA o a los ARAll (la-Altamente recomendado) (recomendación adecuada o de primera línea $)^{88}$.

- Otros antiHTA (alfabloqueadores, betabloqueadores, diuréticos ahorradores de potasio, agentes de acción central, combinación alfa- y betabloqueadores, y vasodilatadores directos) deben reservarse para niños que no responden a dos o más de los agentes preferidos (la-Altamente recomendado) (recomendación adecuada o de primera línea $)^{79}$.

- Los IECA y los ARAll, están contraindicados en pacientes con falla renal aguda, estenosis bilaterales de arteria renal, pacientes con riñón único con estenosis de arteria 
renal (la-Altamente recomendado) (recomendación adecuada o de primera línea) $)^{79,82,93,94}$.

- Los IECA y los ARAll, están contraindicados en el embarazo porque pueden causar lesiones y/o la muerte del feto, por lo que en adolescentes en edad fértil se pueden considerar los bloqueadores de los canales de calcio y los betabloqueadores, siempre y cuando no existan contraindicaciones para su uso (la-Altamente recomendado) (recomendación adecuada o de primera línea) ${ }^{82,84,90,95}$.

- Los betabloqueadores no cardioselectivos deben evitarse en pacientes con asma moderada a severa o en pacientes con enfermedad pulmonar crónica debido a un mayor riesgo de broncoespasmo (la-Altamente recomendado) (recomendación adecuada o de primera línea) $^{82,84,90,95}$.

\section{Pregunta 11 ¿Qué debe saber el médico acerca de la hipertensión arterial secundaria?}

- Se debe realizar una aproximación dirigida por el examen físico, rango etario y posibilidades clínicas según fenotipo del paciente para minimizar estudios extensos y dispendiosos que llevan a manejos y aproximaciones erróneas (la-Altamente recomendado) (recomendación adecuada o de primera línea) ${ }^{96,97}$.

- Se debe tener en cuenta que los pacientes con hipertensión arterial resistente son más propensos a tener hipertensión arterial secundaria y por tanto en este grupo de pacientes se recomiendan estudios dirigidos al diagnóstico de esta enfermedad. El conocimiento de las enfermedades causantes de hipertensión arterial secundaria es obligatorio para aproximar el diagnóstico y el manejo (la-Altamente recomendado) (recomendación adecuada o de primera línea) ${ }^{96,97}$.

- A todo paciente (especialmente hombres con IMC $>35 \mathrm{~kg} / \mathrm{m}^{2}$ ) con hipersomnia diurna asociada a hipertensión arterial mal controlada o severa non dipper se le debe descartar SAHOS como causal secundaria (la-Altamente recomendado) (recomendación adecuada o de primera línea) $)^{98-102}$.

- En todo paciente con SAHOS con evidencia de compromiso cardiaco por ecocardiograma y que no está diagnosticado como hipertenso se debe realizar seguimiento con el fin de determinar si hay hipertensión arterial asociada (laAltamente recomendado) (recomendación adecuada o de primera línea $)^{98-102}$.

- En todo paciente con hipertensión arterial mal controlada con elevación de la creatinina por encima de $1,5 \mathrm{mg} / \mathrm{dl}$ asociado a alteraciones en el sedimento urinario $\mathrm{y} / \mathrm{o}$ alteraciones del parénquima renal en la ultrasonografía renal, debe hacer sospechar enfermedad parenquimatosa renal como causa de hipertensión arterial secundaria. Este paciente debe ser dirigido a especialistas en enfermedades renales para su manejo (la-Altamente recomendado) (recomendación adecuada o de primera línea) ${ }^{103-105}$.

- En pacientes mayores de 50 años con alta carga aterosclerótica asociada a factores de riesgo cardiovascular se debe sospechar enfermedad renovascular aterosclerótica como causa de hipertensión arterial secundaria (la-Altamente recomendado) (recomendación adecuada o de primera línea) $)^{103-105}$.

- En adultos jóvenes (< 40 años), mujeres y niños con hipertensión arterial resistente se debe descartar displasia fibromuscular como causa del mal control de la presión arterial (la-Altamente recomendado) (recomendación adecuada o de primera línea) ${ }^{107-109}$.

- En pacientes hipertensos menores de 40 años con hipertrofia de ventrículo izquierdo sin otra explicación asociado a hipocalemia (con o sin uso de diuréticos), se debe buscar hiperaldosteronismo primario (la-Altamente recomendado) (recomendación adecuada o de primera línea) $)^{109,110}$.

- En caso de sospecha de hiperaldosteronismo primario o hipertensión arterial resistente el medicamento a añadir es la espironolactona (la-Altamente recomendado) (recomendación adecuada o de primera línea) ${ }^{111,112}$.

- Se debe estudiar coartación de aorta como causa de hipertensión arterial en pacientes jóvenes asociado a virus de la inmunodeficiencia adquirida, pulsos disminuidos en miembros inferiores en comparación con pulsos superiores y/o gradiente de presión > $20 \mathrm{~mm} \mathrm{Hg}$ entre los miembros superiores e inferiores (la-Altamente recomendado) (recomendación adecuada o de primera línea) ${ }^{113}$.

- En pacientes con alta sospecha clínica de feocromocitoma (hipertensión arterial episódica en combinación con paroxismos de palpitaciones, hiperhidrosis, palidez y cefalea palpitante) se debe hacer tamizaje con metanefrinas en orina; dada la baja frecuencia de esta enfermedad no recomendamos realizarla de primera línea (la-Altamente recomendado) (recomendación adecuada o de primera línea) $)^{114-116}$.

- Para definir hipertensión arterial resistente se debe descartar como primera medida hipertensión arterial pseudosevera. Para esto se recomiendan los siguientes pasos $^{103}$ :

1. Asegúrese que está tomando la tensión arterial con el método adecuado y que no hay fallos en la toma.

2. Siempre pregúntese si su paciente tiene buena adherencia al tratamiento. La mala adherencia es casi del $42 \%$ en los hipertensos.

3. Un 15 a un $30 \%$ de los pacientes de su consulta presentan hipertensión de bata blanca. En ese caso se recomienda el MAPA o una automonitorización de la presión arterial ambulatoria (AMPA) para diagnóstico de hipertensión con miras a dar manejo terapéutico si se requiere.

4. Pregunte a su paciente sobre posibles medicamentos que pueden aumentar la tensión arterial. No olvide que hay combinaciones de antiHTA que paradójicamente pueden aumentar la presión arterial si se combinan de manera inadecuada.

- Para definir hipertensión arterial resistente debe tener tres medicaciones antiHTA de los grupos básicos y uno de estos debe ser un diurético a dosis plenas ${ }^{103}$.

- El medicamento a añadir en pacientes con hipertensión arterial resistente es la espironolactona ${ }^{103}$. 
Pregunta 12 ¿Cuáles son las indicaciones actuales de manejo intervencionista para los pacientes con hipertensión arterial y cuáles son aplicables en Colombia?

- El manejo intervencionista para los pacientes con hipertensión arterial adquiere importancia en el escenario de la hipertensión arterial resistente, la cual se convierte en un desafío clínico verdadero, que aumenta las complicaciones cardiovasculares de la enfermedad. La estenosis aterosclerótica de las arterias renales es la principal causa de hipertensión arterial renovascular. Se asocia a hipertensión resistente, insuficiencia renal y edema agudo de pulmón. La terapia de revascularización percutánea con balón y/o stent renal en estos casos es controversial (IIIrecomendadas solo si el criterio clínico ve utilidad) ${ }^{117-119}$.

- Teniendo en cuenta que la evidencia no demuestra beneficio de la terapia de revascularización sobre el manejo médico en el tratamiento de la hipertensión arterial resistente en pacientes con estenosis de las arterias renales, este tratamiento no lo recomienda de rutina la guía de la Sociedad Europea de Cardiología (III-recomendadas solo si el criterio clínico ve utilidad) $)^{117-119}$.

- La cirugía debe reservarse para el tratamiento de estenosis asociadas con aneurismas complejos, lesiones complejas o fracaso del tratamiento endovascular (IlbRecomendado) (recomendación adecuada o de primera línea) $)^{117-120}$.

- En caso de edema pulmonar agudo, insuficiencia cardiaca congestiva con función ventricular izquierda conservada o insuficiencia renal aguda oligoanúrica, a pesar de los resultados controversiales puede estar indicada la revascularización endovascular como terapia coadyuvante (Ilb-recomendado) (recomendación adecuada o de primera línea $)^{121}$.

- El tratamiento endovascular para los pacientes con displasia fibromuscular (compromiso renal) está reservado para aquellos con hipertensión arterial resistente y/o signos de disfunción renal. La primera línea de manejo es la angioplastia con balón (Ilb-Recomendado) (recomendación adecuada o de primera línea). El uso de stent solo está indicado en caso de disección de la arteria renal o fallo de la terapia con angioplastia, en tanto que la cirugía se reserva sólo para casos extremos (Ilb-Recomendado) (recomendación adecuada o de primera línea) ${ }^{122,123}$.

- La guía del Colegio Americano de Cardiología y la Sociedad Americana del Corazón para la prevención, detección, evaluación y manejo de la presión arterial elevada en adultos considera que no hay evidencia para recomendar la terapia de denervación renal en pacientes con hipertensión arterial resistente (III-recomendadas solo si el criterio clínico ve utilidad $)^{4}$.

\section{Pregunta 13 ¿Cómo debe ser la valoración prequirúrgica y el manejo perioperatorio del paciente hipertenso?}

- Al enfrentar al paciente hipertenso de cara a una intervención quirúrgica se plantean algunas cuestiones básicas, a saber (la-Altamente recomendado) (recomendación adecuada o de primera línea) $)^{124}:</$ ce:para $></$ ce:listitem $></$ ce:list $>$

- ¿Qué riesgo presenta la anestesia en el paciente hipertenso?

- ¿Qué pruebas complementarias son necesarias para evaluar el grado de afectación y riesgo del paciente hipertenso?

- ¿Qué medicación antihipertensiva es adecuada para el paciente hipertenso que se va a intervenir quirúrgicamente?

- ¿Cuándo ha de posponerse la intervención quirúrgica en el paciente hipertenso mal controlado?

- ¿Cuál debe ser el manejo del paciente hipertenso en el perioperatorio?

En cuanto a la inminencia del procedimiento, es posible clasificar las cirugías como (la-Altamente recomendado) (recomendación adecuada o de primera línea) ${ }^{124}$ :

- Emergencia médica: riesgo inminente de muerte o pérdida de un miembro si no se interviene antes de seis horas.

- Urgencia médica: puede haber tiempo para una evaluación médica limitada, pues la vida o el miembro amenazado pueden perderse en el curso de 6 a 24 horas.

- Procedimiento sensible al tiempo: en este caso la demora en realizar el procedimiento entre una y seis semanas, causará un desenlace negativo.

- Cirugía electiva: el procedimiento puede ser retrasado hasta por un año.

La importancia de esta diferenciación radica en que, en caso de una emergencia, cualquier preocupación acerca de las cifras tensionales pasa a un segundo lugar, y se procede con el acto operatorio sin tardanza, mientras que, por el contrario, una cirugía electiva da tiempo de buscar el mejor control posible de la presión arterial antes de su ejecución (la-Altamente recomendado) (recomendación adecuada o de primera línea) $)^{124}$.

Los factores que dependen del estado del individuo y que determinan un mayor riesgo cardiovascular, tal y como se ha mencionado, no dependen tanto de las cifras de presión arterial como de las enfermedades asociadas. Estos son (la-Altamente recomendado) (recomendación adecuada o de primera línea) ${ }^{124-127}$.

- Factores mayores: síndromes coronarios agudos inestables (infarto agudo o reciente del miocardio con isquemia residual, angina inestable con clase funcional III-IV), insuficiencia cardíaca congestiva descompensada, arritmias significativas (bloqueo AV avanzado, arritmia ventricular en presencia de cardiopatía estructural, arritmia supraventricular con frecuencia cardíaca elevada), estenosis aórtica grave (gradiente medio $\geq 40 \mathrm{~mm} \mathrm{Hg}$, área valvular $\leq$ de $1 \mathrm{~cm}^{2}$, o sintomática).

- Factores intermedios: angina de pecho de clase funcional $1 \circ 2$, historia clínica de infarto del miocardio previo, historia de falla cardíaca previa, diabetes mellitus, insuficiencia renal crónica (depuración de creatinina menor de $60 \mathrm{ml} / \mathrm{min}$ o albuminuria mayor de $30 \mathrm{mg} / \mathrm{g}$ de creatinina) y enfermedad vascular cerebral.

- Factores menores: edad mayor de 70 años, electrocardiograma (EKG) anormal, bien sea por hipertrofia ventricular izquierda, bloqueo de rama izquierda, 
anormalidades del segmento ST, arritmia no sinusal, hipertensión no controlada.

- Dos indicadores adicionales que deben tenerse en cuenta tienen que ver con el estado general de la persona, definidos por la clase funcional y la fragilidad, que determinarán la capacidad del individuo ante la situación estresante que implica el acto quirúrgico (laAltamente recomendado) (recomendación adecuada o de primera línea) ${ }^{126}$.

- La clase funcional se determina mediante el uso de cuestionarios sencillos ya estandarizados que determinan el consumo de oxígeno (equivalentes metabólicos o MET) de ciertas actividades físicas usuales. La capacidad funcional será excelente si es mayor a $10 \mathrm{MET}$, buena si está entre 7 y 10 MET, moderada si está entre 4 y 6 MET, y pobre si es menor. Una capacidad funcional menor a 4 MET permite diferenciar a los pacientes con mayores posibilidades de desarrollar complicaciones cardiovasculares asociadas al procedimiento quirúrgico (la-Altamente recomendado) (recomendación adecuada o de primera línea) ${ }^{126}$.

- No existe un esquema fijo de evaluación clínica ni hay un paquete de paraclínicos estándar. Si se ha determinado que el paciente hipertenso es de bajo riesgo por su estado general y el tipo de cirugía, entonces no requerirá paraclínicos distintos de electrocardiograma, pruebas de función renal, electrolitos, glicemia en ayunas, hemograma y pruebas de coagulación (laAltamente recomendado) (recomendación adecuada o de primera línea) ${ }^{127}$.

- En la medida que el riesgo se vaya haciendo mayor, y siempre de manera orientada, se podrá solicitar ecocardiograma, pruebas para detectar isquemia miocárdica o monitorización electrocardiográfica de 24 horas (Holter) (la-Altamente recomendado) (recomendación adecuada o de primera línea) ${ }^{124}$.

- Nunca en caso de una emergencia quirúrgica los paraclínicos deberán retrasar la inmediatez del procedimiento quirúrgico, mientras que la cirugía electiva permite un rango mayor de estudio, pero no sin una justificación adecuada, determinada por los factores antes mencionados (la-Altamente recomendado) (recomendación adecuada o de primera línea) ${ }^{124}$.

- En el caso del paciente que viene en manejo con diuréticos, estos se deben mantener hasta el día de la cirugía, y el tratamiento por vía oral debe reanudarse tan pronto como sea posible. En el prequirúrgico deben medirse los niveles séricos de electrolitos por el riesgo de hipocalemia en el caso del uso de diuréticos tiazídicos y similares a tiazidas (hidroclorotiazida, clortalidona, indapamida) o hipercalemia si se está usando espironolactona o eplerenona. Esto es especialmente importante si hay uso concomitante de antagonistas de la angiotensina o IECA. Otro riesgo potencial es la depleción de volumen, la potenciación de los relajantes musculares, la hipotensión y el íleo paralítico (laAltamente recomendado) (recomendación adecuada o de primera línea) ${ }^{127}$.

- En cuanto al uso de betabloqueadores, existe una controversia aún no resuelta si estos son o no benéficos en el contexto de la cirugía, con estudios que muestran incremento de la mortalidad, y otros que indicarían un claro beneficio de su uso en el trasquirúrgico a fin de disminuir eventos cardiovasculares y muerte. Sin embargo, hay fuerte cuestionamiento de los estudios que muestran favorabilidad por sesgos múltiples y sospecha de que los resultados pudieran haber sido influenciados por los investigadores, por lo cual no se puede, al día de hoy, indicar su uso profiláctico en cirugía. El paciente que los viene tomando debe continuarlos hasta el día de la cirugía, y se prestará especial atención a la presencia de bradiarritmias, bloqueos e hipotensión (la-Altamente recomendado) ${ }^{128}$.

- Respecto a los calcioantagonistas, se sugiere usarlos hasta el día de la cirugía. Un metaanálisis de varios estudios realizados con un total de 1.007 pacientes, encontró que su uso disminuye el riesgo de arritmias supraventriculares, infarto y falla cardíaca, pero pareciera que los resultados pudieran ser solo atribuibles al uso de diltiazem (la-Altamente recomendado) (recomendación adecuada o de primera línea) ${ }^{129}$.

- Si bien cada día el uso de alfa 2 agonistas es menos frecuente, aún es significativo el número de pacientes que los toman. No se recomienda retirarlos en el día previo, pues hay alto riesgo de hipertensión de rebote. Se debe tener en cuenta que aumentan el riesgo de hipotensión y bradicardia perioperatoria. Su uso como profilácticos no previene la mortalidad ni disminuye los eventos cardiovasculares (la-Altamente recomendado) (recomendación adecuada o de primera línea) ${ }^{130}$.

- En caso de que se presente hipertensión severa durante la cirugía, sabiendo como se ha dicho que estos picos aumentan la morbimortalidad, deben ser controlados pronto, tratando de mantener la presión arterial entre el 70 y el $100 \%$ de la cifra basal, evitando la taquicardia. Se deben manejar medicamentos parenterales, de acción corta y fácil titulación, preferiblemente en forma de infusión continua (la-Altamente recomendado) (recomendación adecuada o de primera línea) ${ }^{125}$.

- Dentro de este contexto, el medicamento más favorable sería el esmolol, el cual es un betabloqueador de acción corta, cardioselectivo y cuyo metabolismo es independiente de la función hepática o renal. Se inicia con un bolo de 250 a $500 \mathrm{mcg} / \mathrm{kg}$ en 1 minuto. El bolo se puede repetir en 5 minutos, con infusión de mantenimiento de $150 \mathrm{mcg} / \mathrm{kg} / \mathrm{min}$. En caso de hipotensión secundaria, la suspensión del efecto al cerrar la infusión se tendrá a los 10 minutos (la-Altamente recomendado) (recomendación adecuada o de primera línea) ${ }^{125}$.

- Otro medicamento que se puede usar en infusión en el transquirúrgico es el nitroprusiato de sodio; sin embargo, se debe tener en cuenta que aumenta la presión intracraneana y que puede producir robo coronario. La dosis es de 0,25 a $10 \mathrm{mcg} / \mathrm{kg} / \mathrm{min}$; el inicio de acción es casi inmediato y dura hasta 2 minutos luego de suspender la infusión (la-Altamente recomendado) (recomendación adecuada o de primera línea) ${ }^{127}$.

- En caso de hipertensión y edema pulmonar por insuficiencia cardíaca o si hay isquemia miocárdica asociada se indica el uso de nitroglicerina en infusión a dosis de 5 a $100 \mathrm{mcg} / \mathrm{min}$. Por reducir la precarga tiende a producir bajo gasto, efecto indeseable si hay hipoperfusión cerebral o renal (la-Altamente recomendado) (recomendación adecuada o de primera línea) ${ }^{127}$. 

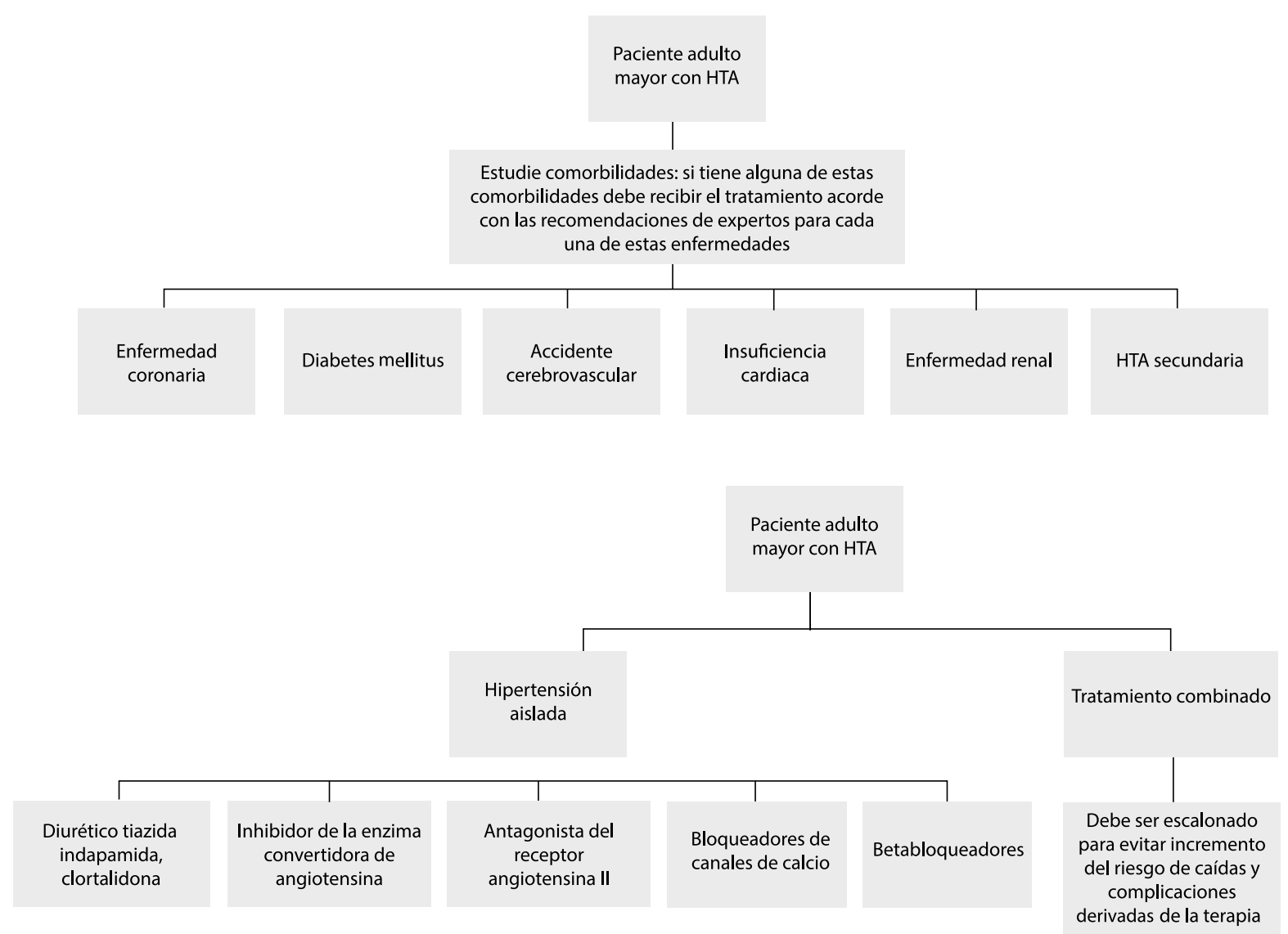

Figura 1 Manejo del paciente adulto mayor con hipertensión arterial

- Como adyuvante en la hipertensión asociada a falla cardíaca y edema pulmonar se indica la furosemida en bolos endovenosos (la-Altamente recomendado) (recomendación adecuada o de primera línea) ${ }^{127}$.

\section{Pregunta 14 ¿Cuál es el tratamiento de la hipertensión en el adulto mayor?}

- El tratamiento antihipertensivo del adulto mayor requiere un abordaje integral, por las comorbilidades y la polifarmacia del paciente y que, a pesar de la edad, los adultos mayores no tienen que ser diferentes del adulto menor de 65 años, de hecho, no hay ningún estudio de pacientes mayores de 65 años que haya demostrado menos beneficio en la reducción de cifra tensionales en comparación a adultos jóvenes (la-Altamente recomendado) (recomendación adecuada o de primera línea $)^{4,127}$.

- En el momento de elegir la terapia antihipertensiva en pacientes adultos mayores se recomienda seguir el algoritmo que se representa como figura 1.

La recomendación de metas para un adulto mayor con hipertensión arterial es $<140 \mathrm{~mm} \mathrm{Hg}$ de presión arterial sistólica siempre y cuando la presión diastólica no sea inferior a $80 \mathrm{~mm} \mathrm{Hg}$. Si el paciente es considerado de fragilidad considerable se puede admitir una meta menor de $150 \mathrm{~mm} \mathrm{Hg}$. (lla-recomendado) (recomendación adecuada o de primera línea) $)^{4,127-129}$.
En cuanto al tratamiento en pacientes con enfermedad renal o diabetes o insuficiencia cardiaca, debe orientarse con base en las metas y los objetivos terapéuticos preespecificados para cada enfermedad en metas de prevención secundaria. Por el contrario, en aquellos pacientes con comorbilidades altas y baja expectativa de vida, el juicio clínico debe prevalecer e individualizar el esquema de tratamiento (Ila-recomendado) (recomendación adecuada o de primera línea $)^{4,127-129}$.

\section{Conflicto de interés}

Ninguno.

\section{Agradecimientos}

A Laboratorios Pfizer y Abbott, así como a la Liga Colombiana contra el Infarto y la Hipertension Arterial.

A la Sociedad Colombiana de Cardiología, Capítulo de Hipertensión Arterial.

\section{Bibliografía}

1. US Burden of Disease Collaborators. The State of US Health, 1990-2010: Burden of Diseases, Injuries, and Risk Factors. JAMA. 2013;310:591-608, http://dx.doi.org/10. 1001/jama.2013.13805. 
2. Mills KT, Bundy JD, Kelly TN, et al. Global Disparities of Hypertension Prevalence and Control: A Systematic Analysis of Population-based Studies from 90 Countries. Circulation. 2016;134:441-50, http://dx.doi.org/10. 1161/CIRCULATIONAHA.115.018912.

3. Forouzanfar M, Liu P, Roth G, et al. Global Burden of hypertension and systolic blood pressure of at least 110 to $115 \mathrm{~mm}$ Hg, 1990-2015. JAMA. 2017;317:165-82, http://dx.doi. org/10.1001/jama.2016.19043.

4. Age-specific relevance of usual blood pressure to vascular mortality: a meta-analysis of individual data for one million adults in 61 prospective studies. The Lancet. Volume 360 , Issue 9349, 2002, Pages 1903-1913,ISSN 0140-6736, https://doi. org/10.1016/S0140-6736(02)11911-8.

5. [2017] ACC/AHA/AAPA/ABC/ACPM/AGS/APhA/ASH/ASPC/ NMA/PCNA Guideline for the Prevention, Detection, Evaluation, and Management of High Blood Pressure in Adults: A Report of the American College of Cardiology/American Heart Association Task Force on Clinical Practice Guidelines. J Am Coll Cardiol 2018;71: e127-e248.

6. James PA, Oparil S, Carter BL, et al. 2014 Evidence-Based Guideline for the Management of High Blood Pressure in AdultsReport from the Panel Members Appointed to the Eighth Joint National Committee (JNC 8). JAMA. 2014;311:507-20, http://dx.doi.org/10.1001/jama.2013.284427.

7. Qaseem A, Wilt TJ, Rich R, et al. Pharmacologic Treatment of Hypertension in Adults Aged 60 Years or Older to Higher Versus Lower Blood Pressure Targets: A Clinical Practice Guideline from the American College of Physicians and the American Academy of Family Physicians. Ann Intern Med.; 166:430-437. doi: 10.7326/M16-1785.

8. Muntner P, Carey R, Gidding S, et al. Potential US Population Impact of the 2017 ACC/AHA High Blood Pressure Guideline. Circulation. 2017;137:109-18.

9. Bundy JD, Mills KT, Chen J, et al. Estimating the Association of the 2017 and 2014 Hypertension Guidelines with Cardiovascular Events and Deaths in US AdultsAn Analysis of National Data. JAMA Cardiol. 2018;3:572-81, http://dx.doi.org/10.1001/jamacardio.2018.1240.

10. Sánchez Pedraza R, Jaramillo González LE. Metodología de calificación y resumen de las opiniones dentro de consensos formales. rev. colomb. psiquiatr [Internet]. 2009 Dec;38:777-86 [cited 2018 Sep 28].

11. MacMahon S, Peto R, Collins R, et al. Blood pressure, stroke, and coronary heart disease: Part 1, prolonged differences in blood pressure: prospective observational studies corrected for the regression dilution bias. The Lancet. 1990;335: 765-74.

12. Hannson L, Zanchettu A, Carruthers G, et al. Effects of intensive blood-pressure lowering and low-dose aspirin in patients with hypertension: principal results of the Hypertension Optimal Treatment (HOT) randomised trial. The Lancet. 1998:351:1755-62.

13. The Blood Pressure Lowering Treatment Trialists' Collaboration. Blood pressure-lowering treatment based on cardiovascular risk: a meta-analysis of individual patient data. The Lancet. 2014;384:591-8.

14. Blood pressure-lowering treatment based on cardiovascular risk: a meta-analysis of individual patient data. The Lancet. 2014;384(9943):591-8.

15. Beckett N, Peters R, Fletcher A, et al. Treatment of Hypertension in Patients 80 Years of Age or Older. N Engl J Med. 2008;358:1887-98.

16. The, SPRINT., Research Group. A Randomized Trial of Intensive versus Standard Blood-Pressure Control. New Eng J Med. 2015;373:2103-16, http://dx.doi.org/10.1056/ NEJMoa1511939.
17. Xie X, Atkins E, Jicheng LV, et al. Effects of intensive blood pressure lowering on cardiovascular and renal outcomes: updated systematic review and meta-analysis. The Lancet. 2016;387(10017):435-43.

18. The, ACCORD., Study Group. Effects of Intensive BloodPressure Control in Type 2 Diabetes Mellitus. N Engl J Med. 2010;362:1575-85.

19. Atkins E, Rodgers A. More versus less blood pressure lowering: an update. clinical therapeutics. 2016;38:213541.

20. Sim JJ, Shi J, Kovesdy CP, et al. Impact of achieved blood pressures on mortality risk and end-stage renal disease among a large, diverse hypertension population. J Am Coll Cardiol. 2014;64:588-97.

21. Haider AW, Larson MG, Franklin SS, et al. Framingham Heart Study. Systolic blood pressure, diastolic blood pressure, and pulse pressure as predictors of risk for congestive heart failure in the Framingham Heart Study. Ann Intern Med. 2003;138:10-6.

22. Yusuf S, Hawken S, Ôunpuu S, et al. Effect of potentially modifiable risk factors associated with myocardial infarction in 52 countries (the INTERHEART study): case-control study. Lancet. 2004:937-52.

23. Roberts A. Risk factors: Effects of alcohol consumption on MI risk-evidence from INTERHEART. Nat Rev Cardiol. 2014;11:434, http://dx.doi.org/10.1038/nrcardio.2014.94. Epub 2014 Jul 1.

24. Piepoli MF, Hoes AW, Agewall S, et al. Guía ESC 2016 sobre prevención de la enfermedad cardiovascular en la práctica clínica. Rev Esp Cardiol. 2016;69:939, e1-e87.

25. NICE. Cardiovascular risk assessment and lipid modification. 2015. Disponible en: https://www.nice.org.uk/ guidance/qs100.

26. Ronald C. Systemic hypertension mechanisms and diagnosis. En: Zipes DP, Libby P, Bonow RO, Mann DL, Tomaselli GF, Braunwald $\mathrm{E}$, editores. Braunwald's heart disease: a textbook of cardiovascular medicine. Elsevier; 2018.

27. Franklin SS, Thijs L, Asayama K, et al. The cardiovascular risk of white-coat hypertension. J Am Coll Cardiol. 2016;68:2033-43.

28. Pickering TG, James GD, Boddie C, Harshfield GA, Blank $\mathrm{S}$, Laragh JH. How Common Is White Coat Hypertension? JAMA. 1988;259:225-8, http://dx.doi.org/ 10.1001/jama.1988.03720020027031.

29. Stergiou GS, Salgami EV, Tzamouranis DG, et al. Masked hypertension assessed by ambulatory blood pressure versus home blood pressure monitoring: is it the same phenomenon? Am J Hypertens. 2005; 18:772-8.

30. MacDonald T. Home blood pressure monitoring. ECR. 2015;10.

31. Hond ED, Celis H, Fagard R, et al. THOP investigators. Selfmeasured versus ambulatory blood pressure in the diagnosis of hypertension. J Hypertens. 2003 Apr;21:717-22.

32. Warren RE, Marshall T, Padfield PL, et al. Variability of office, 24-hour ambulatory, and self-monitored blood pressure measurements. British J General Practice. 2010;60:675-80, http://dx.doi.org/10.3399/bjgp10X515403.

33. Roush G, Ernst M, Kostis J, et al. head-to-head comparisons of hydrochlorothiazide with indapamide and chlorthalidone. Hypertension. 2015;65:1041-6.

34. López-Jaramillo P, Coca A, Sánchez R, et al. Latin American Society of Hypertension. Hypertension Guidelines: Is It Time to Reappraise Blood Pressure Thresholds and Targets? Position Statement of the Latin American Society of Hypertension. Hypertension. 2016;68:257-62.

35. Rochlani Y, Khan MH, Banach M, et al. Are two drugs better than one? A review of combination therapies for hypertension. Expert Opin Pharmacother. 2017;18:377-86. 
36. Wachholz PA, Masuda PY, Ferrari AC, et al. Factors related to blood pressure control in a prospective cohort of hypertensive outpatients. Acta Scientiarum. Health Sciences. 2016;38:57-63.

37. Xie X, Atkins E, Jicheng LV, et al. Effects of intensive blood pressure lowering on cardiovascular and renal outcomes: updated systematic review and meta-analysis. Lancet. 2018;387(10017):435-43.

38. The SPRINT Research Group. A Randomized Trial of Intensive versus Standard Blood-Pressure Control. N Engl J Med. 2015;373:2103-16.

39. Opie L, Gersh B. Beta-blocking agents. En: Opie LH, Gersh B, editores. Drugs for the Heart. Philadelphia, Pensylvania: W.B. Saunders Company; 2005.

40. Lopatowska P, Mlodawska E, Tomaszuk-Kazberuk A, et al. Adhering to the principles of clinical pharmacology - the correct fixed combinations of antihypertensive drugs. Expert Rev Clin Pharmacol. 2018;11:165-70.

41. ONTARGET Investigators. Telmisartan, ramipril, or both in patients at high risk for vascular events. N Engl J Med. 2008;358:1547-59.

42. Hansson L, Hedner T, Lund-Johansen P, et al. Randomised trial of effects of calcium antagonists compared with diuretics and beta-blockers on cardiovascular morbidity and mortality in hypertension: The Nordic Diltiazem (NORDIL) study. The Lancet. 2000;356:359-65.

43. Nissen SE, Tuzcu EM, Libby $P$, et al. CAMELOT Investigators. Effect of antihypertensive agents on cardiovascular events in patients with coronary disease and normal blood pressure: the CAMELOT study: a randomized controlled trial. JAMA. 2004;292:2217-25.

44. Wu HY, Huang JW, Lin HJ, et al. Comparative effectiveness of renin-angiotensin system blockers and other antihypertensive drugs in patients with diabetes: systematic review and bayesian network meta-analysis. BMJ. 2013;347:f6008.

45. Ettehad D, Emdin CA, Kiran A, et al. Blood pressure lowering for prevention of cardiovascular disease and death: a systematic review and meta-analysis. Lancet-. 2016;387:957-67.

46. Bundy JD, Li C, Stuchlik P, et al. Systolic Blood Pressure Reduction and Risk of Cardiovascular Disease and Mortality: A Systematic Review and Network Meta-analysis. JAMA Cardiol. 2017;2:775-81.

47. Rosendorff C, Lackland DT, Allison M, et al. American College of Cardiology, and American Society of Hypertension. Treatment of hypertension in patients with coronary artery disease. A case-based summary of the 2015. Circulation. 2015;131:e435-70.

48. Vidal-Petiot E, Greenlaw N, Ford I, et al. Relationships Between Components of Blood Pressure and Cardiovascular Events in Patients with Stable Coronary Artery Disease and Hypertension. Hypertension. 2018;71:168-76.

49. Jennings GL. A New Guideline on Treatment of Hypertension in Those with Coronary Artery Disease: Scientific Statement from the American Heart Association, American College of Cardiology, and American Society of Hypertension About Treatment of Hypertension in Patients with Coronary Artery Disease. Heart Lung Circ. 2015;24:1037-40.

50. Wan EYF, Yu EYT, Chin WY, et al. Effect of Achieved Systolic Blood Pressure on Cardiovascular Outcomes in Patients with Type 2 Diabetes: A Population-Based Retrospective Cohort Study. Diabetes Care. 2018;41:1134-41.

51. Gardiner FW, Nwose EU, Bwititi PT, Crockett J, Wang L. Blood glucose and pressure controls in diabetic kidney disease: Narrative review of adherence, barriers and evidence of achievement. J Diabetes Complications. 2018;32:104-12.
52. Knight J, Wong MG, Perkovic V. Optimal targets for blood pressure control in chronic kidney disease: the debate continues. Curr Opin Nephrol Hypertens. 2014;23:541-6.

53. Dojki FK, Bakris GL. Blood pressure control and cardiovascular/renal outcomes. Endocrinol Metab Clin North Am. 2018;47:175-84.

54. Jordan JD, Morbitzer KA, Rhoney DH. Acute treatment of blood pressure after ischemic stroke and intracerebral hemorrhage. Neurol Clin. 2015;33:361-80.

55. Di Stefano C, Vanni E, Mirabella S, et al. Risk factors for arterial hypertension after liver transplantation. J Am Soc Hypertens. 2018:220-9.

56. Reiberger T, Mandorfer M. Beta adrenergic blockade and decompensated cirrhosis. J Hepatol. 2017;66:849-59.

57. Santos M, Shah AM. Alterations in cardiac structure and function in hypertension. Curr Hypertens Rep. 2014;16:428.

58. Devereux RB, Roman MJ. Left ventricular hypertrophy in hypertension: stimuli, patterns, and consequences. Hypertens Res. 1999;22:1-9.

59. Díaz Arrieta G, Mendoza Hernández ME, Hernández Cabrera $\mathrm{J}$, et al. Correlación entre la radiografía de tórax y el ecocardiograma para la valoración de cardiomegalia en pacientes con hipertensión arterial sistémica. Arch. Cardiol. Méx. [revista en la Internet]. 2006 Jun [citado 2018 Sep 29].

60. Cheitlin MD, Armstrong WF, Aurigemma GP, et al. ACC/AHA/ASE 2003 guideline update for the clinical application of echocardiography: summary article: a report of the American College of Cardiology/American Heart Association Task Force on Practice Guidelines (ACC/AHA/ASE Committee to Update the 1997 Guidelines for the Clinical Application of Echocardiography). Circulation. 2003;108: 1146-62.

61. Blessberger H, Binder T. NON-invasive imaging: Two dimensional speckle tracking echocardiography: basic principles. Heart. 2010;96:716-22.

62. Sosa-Rosado JM. Tratamiento no farmacológico de la hipertensión arterial. An. Fac. med. [Internet]. 2010 Dic;71:241-4 [citado 2018 Sep 29].

63. NICE. Hypertension in adults: diagnosis and management. 2011. https: / / www. nice.org.uk/guidance/Cg127.

64. Appel LJ, Moore TJ, Obarzanek E, et al. A clinical trial of the effects of dietary patterns on blood pressure. DASH Collaborative Research Group. N Engl J Med. 1997;336:1117-24.

65. Bibbins-Domingo K, Chertow GM, Coxson PG, et al. Projected effect of dietary salt reductions on future cardiovascular disease. N Engl J Med. 2010;362:590-9.

66. Whelton PK, Appel LJ, Espeland MA, et al. Sodium reduction and weight loss in the treatment of hypertension in older persons: a randomized controlled trial of nonpharmacologic interventions in the elderly (TONE). TONE Collaborative Research Group. JAMA. 1998;279:839-46.

67. Stamler J, Chan Q, Daviglus ML, et al. Relation of Dietary Sodium (Salt) to Blood Pressure and Its Possible Modulation by Other Dietary Factors: The INTERMAP Study. Hypertension. 2018;71:631-7.

68. Aristizábal D, García E, McEwen J, et al. Bases genéticas de la hipertensión arterial esencial en Colombia: avances en nueve años de estudio. Rev. Colomb. Cardiol. [Internet]. 2006 Apr;12:409-30 [cited 2018 Sep 29].

69. Cornelissen VA, Smart NA. Exercise training for blood pressure: a systematic review and meta-analysis. J Am Heart Assoc. 2013;2:e004473.

70. Brook RD, Appel LJ, Rubenfire M, et al. Beyond medications and diet: alternative approaches to lowering blood pressure: a scientific statement from the american heart association. Hypertension. 2013;61:1360-83. 
71. James A, King D, Glisson J, et al. The natural treatment of hypertension. Medscape. Disponible en: https: / / www. medscape.com/viewarticle/478882.

72. Tarver T. The Review of Natural Products. Eighth edition, edited by Ara DerMarderosian and John A. Beutler. St. Louis, MO: Facts \& Comparisons ${ }^{\circledR}$, part of Wolters Kluwer Health, 2014.

73. Rasmussen CB, Glisson JK, Minor DS. Dietary supplements and hypertension: potential benefits and precautions. J Clin Hypertens (Greenwich). 2012;14:467-71.

74. Kumar A, Kaur H, Devi P, Mohan V. Role of coenzyme Q10 (CoQ10) in cardiac disease, hypertension and Meniere-like syndrome. Pharmacol Ther. 2009;124:259-68.

75. Li M, Tjen-A-Looi T, Guo ZL, Longhurst J. Repetitive Electroacupuncture Attenuates Cold-Induced Hypertension through Enkephalin in the Rostral Ventral Lateral Medulla. Scientific Reports volume 6. Article number:. 2016;35791.

76. Zhou W, Longhurst JC. Neuroendocrine mechanisms of acupuncture in the treatment of hypertension. Evid Based Complement Alternat Med. 2012;2012:878673.

77. Kalish LA, Buczynski B, Connell P, et al. Stop Hypertension with the Acupuncture Research Program (SHARP): clinical trial design and screening results. Control Clin Trials. 2004 Feb;25:76-103.

78. Sharma AK, Metzger DL, Rodd CJ. Prevalence and Severity of High Blood Pressure Among Children Based on the 2017 American Academy of Pediatrics Guidelines. JAMA Pediatr. 2018;172:557-65.

79. Flynn JT, Kaelber DC, Baker-Smith CM, et al. Clinical Practice Guideline for Screening and Management of High Blood Pressure in Children and Adolescents. Pediatrics. 2017;140.

80. Dionne JM. Updated Guideline May Improve the Recognition and Diagnosis of Hypertension in Children and Adolescents; Review of the 2017 AAP Blood Pressure Clinical Practice Guideline. Curr Hypertens Rep. 2017;19:84.

81. Samuels J, Bell C, Samuel J, et al. Management of hypertension in children and adolescents. Current Cardiology Reports. 2015;17:107.

82. Bruyne PD. Management of hypertension in children and adolescents. Acta Clin Belg. 2015;70:87-94.

83. Expert. Panel on Integrated Guidelines for Cardiovascular. Expert panel on integrated guidelines for cardiovascular health and risk reduction in children and adolescents: summary report;. 2011.

84. Lopilato AC, Muratagic M, Patel S. Pediatric hypertension: a pharmacological review. AACN Adv Crit Care. 2015;26:81-90.

85. Torrance B, McGuire KA, Lewanczuk R, et al. Overweight, physical activity and high blood pressure in children: a review of the literature. Vasc Health Risk Manag. 2007;3:139-49.

86. American Academy of Pediatrics. National high blood pressure education program working group on high blood pressure in children and adolescents. Pediatrics. 2004.114(Suppl 2), IV-IV.

87. Santi M, Simonetti BG, Leoni-Foglia CF, et al. Arterial hypertension in children. Curr Opin Cardiol. 2015;30:403-10.

88. Dhull RS, Baracco R, Jain A, et al. Pharmacologic Treatment of Pediatric hypertension. Curr Hypertens Rep. 2016;18:32.

89. Bruyne PD, Walle JV. Management of hypertension in children and adolescents. Acta Clin Belg. 2015;70:87-94.

90. Misurac J, Nichols KR, Wilson AC. Pharmacologic management of pediatric hypertension. Pediatr Drugs. 2016;18:31-43.

91. Stern MP. Diabetes and cardiovascular disease. The "common soil"' hypothesis. Diabetes. 1995;44:369-74.

92. Martino F, Puddu PE, Pannarale G, et al. Hypertension in children and adolescents attending a lipid clinic. Eur J Pediatr. 2013;172:1573-9.

93. Bullo M, Tschumi S, Bucher BS, et al. Pregnancy outcome following exposure to angiotensin-converting enzyme inhibitors or angiotensin receptor antagonists: a systematic review. Hypertension. 2012;60:444-50.
94. Flynn JT, Daniels SR, Hayman LL, et al. Update: ambulatory blood pressure monitoring in children and adolescents: a scientific statement from the American Heart Association. Hypertension. 2014;63:1116-35.

95. Rimoldi SF, Scherrer U, Messerli FH. Secondary arterial hypertension: when, who, and how to screen? Eur Heart J. 2014;35:1245-54.

96. Calhoun DA, Jones D, Textor S, et al. Resistant hypertension: diagnosis, evaluation, and treatment: a scientific statement from the American Heart Association Professional Education Committee of the Council for High Blood Pressure Research. Circulation. 2008;117:510-26.

97. Pedrosa RP, Drager LF, Gonzaga CC, et al. Obstructive sleep The most common secondary cause of hypertension associated with resistant hypertension. Hypertension. 2011:811-7.

98. Grote L, Ploch T, Heitmann J, et al. Sleep-related breathing disorder is an independent risk factor for uncontrolled hypertension. J Hypertens. 2000;18:679-85.

99. Lavie P, Hoffstein V. Sleep apnea syndrome: a possible contributing factor to resistant. Sleep. 2001;24:721-5.

100. Bruno RM, Rossi L, Fabbrini M, et al. Renal vasodilating capacity and endothelial function are impaired in patients with obstructive sleep apnea syndrome and no traditional cardiovascular risk factors. J Hypertens. 2013;31:1456-64.

101. Johns MW. Day time sleepiness, snoring, and obstructive sleep apnea. The Epworth Sleepiness Scale. Chest. 1993;03:30-6.

102. Pickering TG, Hall JE, Appel LJ, et al. Recommendations for blood pressure measurement in humans and experimental animals: part 1: blood pressure measurement in humans: a statement for pprofessionals from the Subcommittee of Professional and Public Education of the American Heart Association. Circulation. 2005:697-716.

103. Cloward TV, Walker JM, Farney RJ, et al. Left ventricular hypertrophy is a common echocardiographic abnormality in severe obstructive sleep apnea and reverses with nasal continuous positive airway pressure. Chest. 2003;124:594-601.

104. Aqel RA, Zoghbi GJ, Baldwin SA, et al. Prevalence of renal artery stenosis in high-risk veterans referred to cardiac catheterization. J Hypertens. 2003;21:1157-62.

105. Coresh J, Wei GL, McQuillan G, et al. Prevalence of high blood pressure and elevated serum creatinine level in the United States: findings from the third National Health and Nutrition Examination Survey (1988-1994). Arch Intern Med. 2001:1207-16.

106. Aboyans V, Ricco JB, Bartelink MLE, et al., 2017 ESC Guidelines on the Diagnosis and Treatment of Peripheral Arterial Diseases, in collaboration with the European Society for Vascular Surgery (ESVS) Document covering atherosclerotic disease of extracranial carotid and vertebral, mesenteric, renal, upper and lower extremity arteries Endorsed by: the European Stroke Organization (ESO) The Task Force for the Diagnosis and Treatment of Peripheral Arterial Diseases of the European Society of Cardiology (ESC) and of the European Society for Vascular. Eur Heart J. 2017;39:763-816.

107. Michelis KC, Olin JW, Kadian-Dodov D, et al. Coronary artery manifestations of fibromuscular dysplasia. J Am Coll Cardiol. 2014;64:1033-46.

108. Noilhan C, Barigou M, Bieler L, et al. Causes of secondary hypertension in the young population: A monocentric study. Annales de Cardiologie et d'Angeiologie. 2016;65:159-64.

109. Funder JW, Carey RM, Mantero F, et al. The Management of Primary Aldosteronism: Case Detection, Diagnosis, and Treatment: An Endocrine Society Clinical Practice Guideline. J Clin Endocrinol Metab. 2016:1889-916.

110. Mulatero P, Stowasser M, Loh KC, et al. Increased diagnosis of primary aldosteronism, including surgically correctable 
forms, in centers from five continents. J Clin Endocrinol Metab. 2004;89:1045-50.

111. Calhoun DA, Nishizaka MK, Zaman MA, et al. Hyperaldosteronism among black and white subjects with resistant hypertension. Hypertension. 2002;40:892-6.

112. Dijkema EJ, Leiner T, Grotenhuis HB. Diagnosis, imaging and clinical management of aortic coarctation. Heart. 2017; 103:1148-55.

113. Amar L, Servais A, Gimenez-Roqueplo AP, et al. Year of diagnosis, features at presentation, and risk of recurrence in patients with pheochromocytoma or secreting paraganglioma. J Clin Endocrinol Metabol. 2005;90:2110-6.

114. Lenders JW, Eisenhofer G, Mannelli M, et al. Phaeochromocytoma. The Lancet. 2005;366:665-75.

115. Young WF. Adrenal causes of hypertension: pheochromocytoma and primary aldosteronism. Rev Endocrin Metabol Dis. 2007;8:309-20.

116. Ruilope LM, Arribas F. Hipertensión arterial resistente y denervación renal. Reflexiones tras el estudio Symplicity HTN-3. Rev Esp Cardiol. 2014;67:881-2.

117. Jaff MR, Bates M, Sullivan T, et al. HERCULES Investigators. Significant reduction in systolic blood pressure following renal artery stenting in patients with uncontrolled hypertension: results from the HERCULES trial. Catheterization and Cardiovascular Interventions. 2012;80:343-50.

118. Bavry AA, Kapadia SR, Bhatt DL. Renal artery revascularization: updated meta-analysis with the CORAL trial. JAMA. 2014;174:1849-51.

119. Nordmann AJ, Woo K, Parkes R, et al. Balloon angioplasty or medical therapy for hypertensive patients with atherosclerotic renal artery stenosis? A meta-analysis of randomized controlled trials. Am J Med. 2003;114:44-50.

120. Nieminen M, Böhm M, Cowie M, et al. Guías de Práctica Clínica sobre el diagnóstico y tratamiento de la insuficiencia cardíaca aguda. Versión resumida. Rev Esp Cardiol. 2005;58:389-429.

121. Trinquart L, Mounier-Vehier C, Sapoval M, et al. Efficacy of revascularization for renal artery stenosis caused by fibromuscular dysplasia: a systematic review and meta-analysis. Hypertension. 2010;56:525-32.

122. Persu A, Giavarini A, Touze E, et al. European consensus on the diagnosis and management of fibromuscular dysplasia. J Hypertens. 2014;32:1367-78.
123. Fleisher LA, Beckman JA, Brown KA, et al., ACC/AHA 2007 guidelines on perioperative cardiovascular evaluation and care for noncardiac surgery: a report of the American College of Cardiology/American Heart Association Task Force on Practice Guidelines (writing committee to revise the 2002 guidelines on perioperative cardiovascular evaluation for noncardiac surgery) developed in collaboration with the American Society of Echocardiography, American Society of Nuclear Cardiology, Heart Rhythm Society, Society of Cardiovascular Anesthesiologists. J Am Coll Cardiol. 2007;50:e159-242.

124. Sierra P, Galcerán JM, Sabaté S, et al. Documento de consenso sobre hipertensión arterial y anestesia de las Sociedades Catalanas de Anestesiología e Hipertensión Arterial. Rev Esp Anestesiol Reanim. 2009;56:493-502.

125. Kojima G, Iliffe S, Walters K. Frailty index as a predictor of mortality: a systematic review and meta-analysis. Send to Age Ageing. 2018;47:193-200.

126. Kristensen SD, Knuuti J, Saraste A, et al. 2014 ESC/ESA Guidelines on non-cardiac surgery: cardiovascular assessment and management: The Joint Task Force on non-cardiac surgery: cardiovascular assessment and management of the European Society of Cardiology (ESC) and the European Society of Anaesthesiology (ESA). Eur Herat J. 2014;35:2383-431.

127. Fleisher LA, Fleischmann KE, Auerbach AD, et al. 2014 ACC/AHA guideline on perioperative cardiovascular evaluation and management of patients undergoing noncardiac surgery: a report of the American College of Cardiology/American Heart Association Task Force on Practice Guidelines. J Am Coll Cardiol. 2014;64:e77-137.

128. Wijeysundera DN, Beattie WS. Calcium channel blockers for reducing cardiac morbidity after noncardiac surgery: a metaanalysis. Anesthesia \& Analgesia. 2003;97:634-41.

129. Duncan D, Sankar A, Beattie WS, et al. Alpha-2 adrenergic agonists for the prevention of cardiac complications among adults undergoing surgery. Cochrane Database of Systematic Reviews. 2018. 